\author{
Banco de México \\ Documentos de Investigación
}

$\mathrm{N}^{\circ} 2021-09$

\title{
Efectos de Choques No Anticipados en el Ingreso sobre el Consumo en México, 2000-2016
}

\author{
Irving Llamosas-Rosas \\ Banco de México
}

\author{
Erick Rangel González \\ Banco de México
}

Julio 2021

La serie de Documentos de Investigación del Banco de México divulga resultados preliminares de trabajos de investigación económica realizados en el Banco de México con la finalidad de propiciar el intercambio y debate de ideas. El contenido de los Documentos de Investigación, así como las conclusiones que de ellos se derivan, son responsabilidad exclusiva de los autores y no reflejan necesariamente las del Banco de México.

The Working Papers series of Banco de México disseminates preliminary results of economic research conducted at Banco de México in order to promote the exchange and debate of ideas. The views and conclusions presented in the Working Papers are exclusively the responsibility of the authors and do not necessarily reflect those of Banco de México. 


\section{Documento de Investigación 2021-09 \\ Working Paper \\ 2021-09 \\ Efectos de Choques No Anticipados en el Ingreso sobre el Consumo en México, 2000-2016}

\author{
Irving Llamosas-Rosas ${ }^{\dagger}$ \\ Banco de México
}

\author{
Erick Rangel González \\ Banco de México
}

Resumen: Se analizan los cambios en el consumo de los hogares mexicanos ante cambios no esperados en los ingresos, tanto permanentes como transitorios, durante el periodo 2000-2016 siguiendo la metodología desarrollada por Blundell et al. (2008), la cual permite evaluar qué hipótesis sobre la respuesta del consumo es más acorde con la evidencia empírica en México: de ingreso permanente, de mercados completos, o de aseguramiento parcial. Los resultados sugieren la presencia de aseguramiento parcial en el consumo ante choques permanentes en el ingreso a nivel nacional, aunque también son consistentes con la hipótesis de ingreso permanente. Sin embargo, se encuentran diferencias entre regiones al replicar el mismo análisis. En referencia a los choques temporales, el coeficiente del efecto del choque al ingreso sobre el consumo no resulta estadísticamente significativo, a nivel nacional ni regional, lo que sugiere un suavizamiento del consumo por parte de los hogares ante cambios temporales en su ingreso.

Palabras Clave: Impactos al consumo, ingreso, panel sintético JEL Classification: D12, D31, E21

Abstract: Changes on consumption of Mexican households generated by unexpected shocks in income, both permanent and transitory, are analyzed during the period 2000-2016 following the methodology developed by Blundell et al. (2008), which allows to evaluate which hypothesis on the response of consumption is more in line with the empirical evidence in Mexico: permanent income, complete markets, or partial insurance. The results suggest the presence of partial insurance on consumption in the face of permanent income shocks at the national level, although results are also consistent with the permanent income hypothesis. However, differences are found between regions when replicating the same analysis. Regarding temporary shocks, the coefficient of the effect of income shocks on consumption is not statistically significant at the national or regional level, which suggests a consumption smoothing by households in the face of temporary changes in their income.

Keywords: Consumption shocks, income, syntethic panel

\footnotetext{
† Dirección General de Investigación Económica. Correo: illamosasr@banxico.org.mx.

$\ddagger$ Dirección General de Investigación Económica. Correo: erick.rangel@banxico.org.mx.
} 


\section{Introducción.}

¿Cómo absorben las familias mexicanas diferentes choques en el ingreso? ¿Cómo se reflejan en sus patrones de consumo estos choques de naturaleza distinta? En este documento de investigación se analizan los efectos de los cambios permanentes o transitorios en el ingreso sobre el consumo. En particular, se analizan los cambios en el consumo de los hogares mexicanos ante cambios no esperados en los ingresos (permanentes y transitorios) para el caso de México durante el periodo que comprende el año 2000 al 2016. Dicho análisis se realiza tanto a nivel nacional como para cada una de las cuatro regiones del país.

De acuerdo con la literatura, los efectos en el consumo de los hogares ante cambios en sus ingresos, se clasifican según la naturaleza de los choques de los ingresos entre anticipados y no anticipados (Figura 1). A su vez, los cambios no anticipados en el ingreso pueden categorizarse entre choques permanentes y temporales. Por su parte, los cambios anticipados, dado que son esperados por lo general suelen dividirse entre positivos o negativos dependiendo de si se espera un incremento o una reducción de los ingresos respectivamente.

\section{Figura 1. Respuesta del Consumo ante Cambios en el Ingreso}

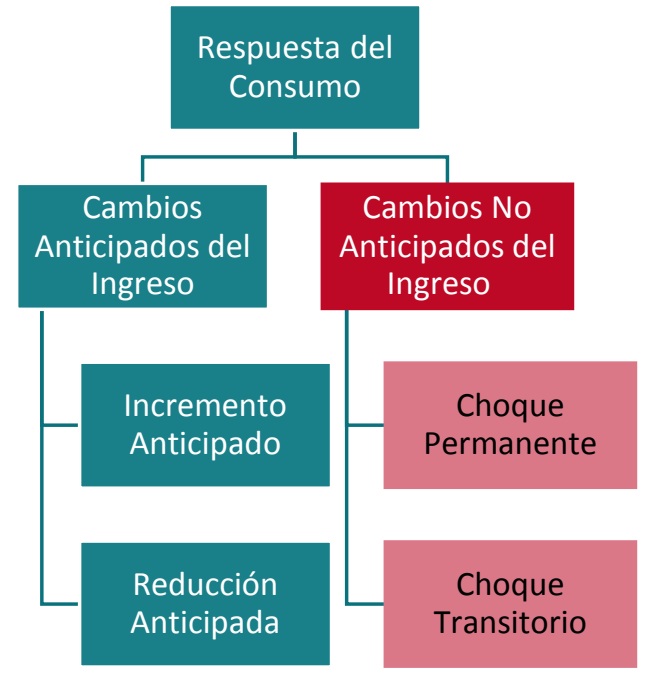

Fuente: Tomada de Jappelli y Pistaferri (2017)

Cuando los cambios en el ingreso son anticipados y no existen restricciones de liquidez, el consumo no debería responder debido a mecanismos de previsión (como el ahorro precautorio, o el uso de líneas de crédito) que los consumidores pueden disponer para 
suavizar su consumo y mantenerlo constante. Sin embargo, cuando existen restricciones en los mercados de crédito, o cuando no se cuenta con mecanismos informales de suavizamiento al consumo como la ayuda de familiares o amigos, es posible observar cambios en el consumo ante cambios anticipados en el ingreso (Jappelli y Pistaferri, 2017).

Por su parte, los choques no anticipados en el ingreso podrían tener efectos en el consumo, los cuales dependerán de las características y duración de estos (Jappelli y Pistaferri, 2010). De acuerdo a la hipótesis del ingreso permanente, los cambios en el consumo serían uno a uno con el ingreso si el choque es permanente, es decir, las familias ajustarían inmediatamente su consumo ante un choque no anticipado y permanente en su ingreso. Sin embargo, los choques transitorios no modificarían el consumo. En contraste, la hipótesis de los mercados completos establece que los choques no anticipados en el ingreso podrían no tener efecto en el consumo de existir mercados crediticios y de seguros completos, debido a que las familias cubrirían cualquier eventualidad con sus ingresos futuros. Un paso intermedio entre estas dos hipótesis es el aseguramiento parcial (no necesariamente formal, sino puede ser por medio de otros mecanismos como apoyo de familiares, transferencias gubernamentales, etc.) en donde un choque no anticipado en el ingreso permanente es cubierto de manera parcial, mientras que los choques transitorios tendrían un efecto moderado.

Siguiendo a Blundell et al. (2008) y Jappelli y Pistaferri (2017), este documento de investigación se centra en el análisis de los cambios en el consumo de los hogares ante cambios no esperados en los ingresos (tanto permanentes como transitorios) para el caso de México durante el periodo que comprende el año 2000 al 2016. Dicho análisis se realiza tanto a nivel nacional como para cada una de las 4 regiones del país. ${ }^{1}$ En específico, el objetivo de esta investigación es probar distintas hipótesis sobre los impactos del consumo ante cambios no esperados en el ingreso en México; en particular la hipótesis del ingreso permanente, que

\footnotetext{
${ }^{1}$ Regionalización: el norte incluye Baja California, Chihuahua, Coahuila, Nuevo León, sonora y Tamaulipas; el centro norte considera Aguascalientes, Baja California Sur, Colima, Durango, Jalisco, Michoacán, Nayarit, San Luis Potosí, Sinaloa y Zacatecas; el centro lo integran la ciudad de México, Estado de México, Guanajuato, Hidalgo, Morelos, Puebla, Querétaro y Tlaxcala; y el sur, Campeche, Chiapas, Guerrero, Oaxaca, Quintana Roo, Tabasco, Veracruz y Yucatán.
} 
establece que choques en el ingreso permanente impactarán por completo al consumo, mientras que los choques temporales tendrían un efecto nulo; la hipótesis de mercados completos, en la cual ni los choques permanentes ni los temporales tienen efecto en el consumo; y la hipótesis de ahorro precautorio o aseguramiento parcial, la cual establece que los hogares suavizan parte de los choques en el ingreso permanente sobre el consumo de las familias.

Para lograr dicho objetivo se emplea una descomposición estadística utilizada en el estudio de Hall y Mishkin (1982) y en Blundell et al. (2008) que permite diferenciar los choques del ingreso entre permanentes y temporales. Para ello, se dispondrá de un panel sintético basado en la Encuesta Nacional de Ingreso y Gasto de los Hogares (ENIGH) organizado por edad del jefe de familia y su nivel educativo, esto con la finalidad de contar con cohortes lo más homogéneas posibles, tanto en el ingreso como en el consumo. Asimismo, dado que en México existe una heterogeneidad importante en los patrones de ingreso y de consumos entre las distintas regiones del país, dicho análisis se replica para las regiones norte, centro norte, centro y sur. Hay que tomar en consideración que el análisis propuesto en este documento de investigación se centra en el gasto promedio de los hogares asociados a las cohortes seleccionadas por el panel dinámico y no precisamente el consumo a nivel de los hogares, debido a la imposibilidad de acceder a un panel del consumo familiar.

Para el caso de los Estados Unidos durante la década de los ochentas Blundell et al. (2008) encuentran aseguramiento parcial en los choques permanentes al ingreso. En específico, por cada 100 dólares de caída en el ingreso permanente no esperado, el consumo de no durables tiene una caída de 64 dólares; dicho aseguramiento parcial es mayor en los hogares con educación superior y cercanos al retiro, y se encuentra aseguramiento completo de los choques transitorios, excepto en hogares pobres.

Existen pocas investigaciones que estudian la relación entre el consumo y los ingresos en México. El documento de investigación de Attanasio y Skékely (1998) analiza la relación del ahorro y el gasto de los hogares en México, principalmente bajo la perspectiva de la crisis financiera de 1994. Encuentra que el ahorro está altamente concentrado en hogares con altos niveles educativos, lo cual puede implicar que están en mejor situación para suavizar 
reducciones en el ingreso después del retiro o suavizar choques temporales en su ingreso. El estudio de Attanasio y Skékely (2004) analiza los efectos en el consumo en los años noventa ante cambios anticipados en el ingreso laboral. Su análisis se enfoca en el impacto de cambios en el ingreso laboral ante varios rubros de consumo a nivel nacional aprovechando el choque negativo en los ingresos de 1995, concluyendo que los hogares de México trasladan los impactos del ingreso al consumo, especialmente en los bienes durables dentro del periodo de análisis. Cabe destacar que dicha aproximación metodológica tiene limitantes, pues no permite diferenciar los cambios en el ingreso temporales de los permanentes.

El presente documento busca contribuir a la literatura para el caso mexicano analizando choques no anticipados en los ingresos tanto temporales como permanentes a nivel nacional y regional. Los resultados sugieren la presencia de aseguramiento parcial en el consumo ante choques permanentes en el ingreso a nivel nacional, aunque también son consistentes con la hipótesis de ingreso permanente. A nivel regional, los coeficientes del efecto del choque permanente en el ingreso sobre el consumo son similares al estimado a nivel nacional en las regiones norte, centro norte y centro, y de mayor magnitud en el sur, si bien con significancia estadística solo en las regiones centro norte y sur. En referencia a los choques temporales, el coeficiente del efecto del choque al ingreso sobre el consumo no resulta estadísticamente significativo a nivel nacional ni regional, lo que sugiere un suavizamiento del consumo por parte de los hogares ante cambios temporales en su ingreso. Si bien, Japelli y Pistaferri (2017) comentan el uso de pseudo datos panel en la literatura, es preciso señalar que los resultados de la presente investigación deben ser interpretados con precaución, debido a que en la construcción del panel sintético existe el potencial de ignorar el diseño muestral. ${ }^{2}$

Los resultados obtenidos en este documento de investigación ofrecen evidencia sobre los efectos que tendría en el consumo, específicamente de no durables, ante un choque no anticipado en el ingreso. Dichos resultados, en línea con la hipótesis de aseguramiento parcial, indican que gran parte de los efectos en el consumo dependen de la perspectiva que se tenga de la duración del choque en el empleo e ingresos de las familias. En otras palabras,

\footnotetext{
${ }^{2}$ La construcción del panel sintético podría no representar de manera fidedigna la distribución poblacional del ingreso y gasto de los hogares.
} 
a mayor expectativa de duración, los efectos en el consumo serán más notorios. Por el otro lado, si los impactos se perciben como temporales, los mecanismos de aseguramiento permitirán que los niveles de consumo no se vean alterados. Esto puede ayudar en entender efectos futuros, como los provocados por el "gran confinamiento del 2020" el cual es un ejemplo claro y específico de un choque no anticipado, si bien no es claro si su duración para algunos hogares será temporal y para otros más permanente.

El documento de investigación está organizado de la siguiente forma: La sección 2 contiene una revisión de literatura sobre las metodologías que analizan los impactos no anticipados del ingreso al consumo. Los datos utilizados para la estimación se describen en la sección 3. El modelo econométrico es explicado en la sección 4, mientras que los resultados se presentan en la sección 5. Finalmente, en la sección 6 se concluye.

\section{Revisión de Literatura}

De acuerdo con Japelli y Pistaferri (2017), existen tres metodologías para evaluar la forma en que el consumo responde ante cambios no anticipados en el ingreso, y la respuesta en estos cambios depende de la persistencia del choque y el grado de las imperfecciones de los mercados crediticios y de seguros.

Un primer método identifica episodios en los cuales los cambios en el ingreso se registraron inesperadamente, por lo cual se hacen estudios cuasi-experimentales de cómo el consumo reacciona a dichos cambios. El primer estudio en realizar dicho análisis es el de Bodkin (1959) utilizando pagos no anticipados hacia los veteranos de guerra de Estados Unidos por parte del National Service Life Insurance Fund (un choque de "una sola vez" en el ingreso, no anticipado por el consumidor). Los resultados contradicen la hipótesis de ingreso permanente, ya que la propensión marginal a consumir varía de 0.7 a 0.9 . En un estudio similar, Kreinin (1961) encuentra un valor más acorde a la hipótesis de ingreso permanente, con un coeficiente de 0.15 . Por su parte, Wolpin (1982) realiza un análisis de panel utilizando choques en las condiciones atmosféricas para los hogares en zonas agrícolas, encontrando una elasticidad del ingreso permanente de 0.91 a 1.02 con respecto al consumo, en línea con la hipótesis del ingreso permanente. Paxon (1993), partiendo del hecho de que el ingreso de 
los hogares de Tailandia depende altamente de la agricultura, analiza su efecto en el consumo, encontrando que la temporalidad de los flujos de ingresos tiene poco que ver con la temporalidad de los gastos.

Gruber (1997) estudia el efecto de los programas gubernamentales de seguro de desempleo en la suavización del consumo en Estados Unidos ante despidos anticipados y no anticipados, encontrando evidencia de efectos de suavizamiento en el consumo en los no anticipados. Para el caso canadiense, Browning y Crossley (2001) encuentran efectos estadísticamente significativos en el suavizamiento del consumo, especialmente en familias con restricciones de liquidez. Finalmente, Agarwal y Quian (2014) analizan mediante datos panel el efecto del anuncio del gobierno de Singapur del "Growth Dividend Program" el cual otorgaba por una sola ocasión a 2.5 millones de ciudadanos un pago que variaba entre \$78 a \$702 dólares por persona. Sus estimaciones concluyen que de cada dólar recibido, se gastaron 80 centavos.

Una segunda aproximación estima el impacto de los choques combinando realizaciones y expectativas de ingreso o consumo en encuestas, en donde datos de expectativas subjetivas están disponibles. Por ejemplo, los consumidores pueden saber con antelación que estarán enfrentando un choque en sus ingresos, pero el evento no es conocido por el investigador; por lo que el choque se tomará como no anticipado, cuando realmente lo es. Para evitar ese problema, los estudios se basan en información cualitativa de los hogares y sus expectativas de ingresos. El estudio de Hayashi (1985) es uno de los primeros en realizar dicho análisis, sus resultados refuerzan la hipótesis de ingreso permanente, con alta sensibilidad del consumo a choques en el ingreso permanente.

La tercera aproximación estima la propensión marginal a consumir con respecto a choques en el ingreso empleando restricciones en las covarianzas que la teoría impone al comportamiento conjunto del crecimiento del consumo e ingreso. El primer artículo en analizar los impactos no anticipados del ingreso al consumo utilizando restricciones en las matrices de covarianza entre el ingreso y el consumo para identificar los parámetros de dichos impactos es el estudio de Hall y Mishkin (1982), el cual asume un impacto en el consumo ante choques permanentes de 1 (en línea con la hipótesis de ingreso permanente) y estima el indicador para los choques temporales, encontrando efectos de $29 \%$. 
Por su parte, Blundell et al. (2008) analizan los efectos en el consumo en Estados Unidos utilizando el Panel Study of Income Dynamics, complementando la muestra de gastos con el Consumer Expenditure Survey, y encuentran aseguramiento parcial de los choques permanentes (con un coeficiente de 0.64 para el consumo de no durables) y aseguramiento completo en choques transitorios (con excepción de hogares pobres, con coeficiente de 0.37 ).

Esta aproximación ofrece ventajas frente a la metodología cuasi-experimental, ya que para usar esta última es necesario un evento generalizado de choque inesperado al ingreso, ${ }^{3}$ y la metodología basada en encuestas no es factible para el caso mexicano. Uno de los retos que tiene la aproximación de restricciones en las covarianzas es la utilización de paneles sintéticos, lo cual se abordará más adelante.

Para el caso de México, el estudio de Attanasio y Skékely (2004) es el que tiene mayor relación con el presente estudio. En él, se estudia el efecto de los choques en el ingreso laboral en México al consumo durante los años noventa. Si bien en este periodo de tiempo se puede argumentar que los cambios no son esperados, la ecuación que se analiza (sensibilidad del consumo ante cambios en el ingreso laboral) se enfoca principalmente a cambios anticipados en el ingreso (a diferencia del enfoque de este documento de investigación). Sus resultados implican que las familias mexicanas tienden a reducir su consumo ante cambios temporales en los ingresos laborales.

\section{Datos}

En el presente documento de investigación se utilizan datos de ingresos y transferencias de la Encuesta Nacional de Ingresos y Gastos de los Hogares para los años 2000, 2002, 2004, 2006, 2008, 2010, 2012, 2014 y 2016, y como indicador de consumo de las familias se emplea el gasto de alimentos y bienes no durables, siguiendo el trabajo de Blundell et al. (2008). A diferencia de la presente investigación, en el trabajo de Blundell fue posible construir un panel de datos de individuos basado en el PSID (Panel Study of Income Dynamics), complementando los datos de consumo de no durables (los cuales no se encuentran en el PSID) con la encuesta CEX (Consumer Expenditure Survey). En el caso de México,

\footnotetext{
${ }^{3}$ Para el cual, el "gran confinamiento" sería un excelente candidato.
} 
contamos con los datos de la ENIGH, que si bien incluyen todo tipo de gastos, no hacen posible observar al mismo individuo a lo largo del tiempo. Debido a lo anterior, abordaremos el problema utilizando paneles sintéticos (pseudo-panel). Dicha aproximación ha sido utilizada por otros autores para atender el mismo problema (Primiceri y van Rens, 2009), quienes en su estudio, construyen un panel sintético con celdas a nivel de edad de la persona de referencia (en este caso, el individuo que es propietario o renta la casa) en cohortes de 10 años para edades de 20 a 65 años, de 1980 al año 2000. Por su parte, Jappelli y Pistaferri (2010) describen el uso en la literatura de pseudo-panel ${ }^{4}$ (sintéticos) para medir los choques en el consumo utilizando dicha descomposición estadística.

En este documento se elaboró un panel sintético siguiendo la propuesta de Attanasio y Skékely (2004), y se utilizaron datos de la Encuesta Nacional de Ingreso y Gasto de los Hogares (ENIGH). Para ello se empleó la clasificación de edad y educación por tratarse de "grupos homogéneos" dentro del panel. ${ }^{5}$ Asimismo, se clasificó el gasto siguiendo a Blundell et al. (2008) en gastos de comida y no durables, ${ }^{6}$ Cabe mencionar que los gastos fueron normalizados por adulto equivalente (Primiceni y van Rens, 2009, Apéndice A), siguiendo la propuesta de Attanasio y Skékely (2004) (basado en el trabajo de Teruel et al., 2005). Primiceni y van Rens (2009), concluye que el consumo de un niño de 0 a 5 años va desde 0.64 hasta 0.77 de un adulto; el de un niño de 6 a 12 años de 0.69 a 0.81 ; y el de un niño mayor entre 13 y 18 va desde 0.62 a $0.76 .^{7}$

Con la finalidad de controlar por las características demográficas que definen al consumo, se tomó en consideración la educación del jefe de familia; número de miembros con escolaridad

\footnotetext{
${ }^{4}$ Jappelli y Pistaferri (2010) comentan la necesidad del uso de datos panel (o pseudo panel) debido la necesidad de tener observaciones para varios periodos de tiempo, tanto de ingreso como de consumo, aunque no necesariamente en la misma base de datos o del mismo hogar.

${ }^{5}$ Debe mencionarse que los cohortes deben capturar las diferencias idiosincráticas principales de los hogares, ya que se asumiría que el comportamiento dentro de los cohortes debe ser homogéneo. En la aproximación de panel sintético, las covarianzas entre los ingresos y consumo explicarían las diferencias entre cohortes.

${ }^{6}$ Para efectos del consumo, se siguió la misma clasificación de consumo que Blundell et al. (2008) con no durables y comida. Cabe destacar que, a diferencia de Blundell et al. (2008) donde el PSID sólo contiene información de consumo de alimentos y, por tanto, deben imputarlo a partir de la encuesta CEX; en nuestro caso la ENIGH sí proporciona suficiente información, por lo que el proceso de imputación no es necesario, aunque la limitante de no tener un panel de individuos, como se verá más adelante, nos lleva a utilizar un panel sintético. En el lado del ingreso, se calculó el ingreso total, incluyendo transferencias de gobierno y remesas.

${ }^{7}$ Para el presente estudio, se tomaron los valores medios de dichas conclusiones.
} 
primaria, media y superior o mayor; la cantidad de menores a 10 años, así como el número de perceptores por familia y las horas trabajadas. ${ }^{8}$

\section{Estadística Descriptiva}

El panel sintético se constituye de 60 individuos característicos en celdas a nivel de grupo de edad y educación. Para los diferentes grupos de edad, se construyeron diez celdas según la fecha de nacimiento de los jefes de familia entre 1941 a 1945 hasta los nacidos entre 1986 a 1990, los cuales tenían entre 25 a 30 años al momento del levantamiento de la ENIGH 2016. En el caso de los distintos grupos educativos, se seleccionó a las familias cuyo jefe de familia tuviese uno de los siete segmentos educativos ${ }^{9}$ en los cuales se dividió la muestra (sin instrucción, de 1 a 4 años de escolaridad, de 5 a 6 años, secundaria terminada, 1er o 2do año de preparatoria, preparatoria terminada, superior o más). Por ejemplo, en la ENIGH 2016, el cohorte con jefe de familia con grado de 5to y 6to de primaria nacidos entre 1971 y 1975 , dispone de 1612 hogares (ver tabla 1).

\footnotetext{
${ }^{8}$ Cabe destacar que si bien los promedios de cohortes propuestas en el panel sintético analizado en este documento de investigación tienen un comportamiento razonablemente similar a los promedios obtenidos con el factor de expansión de los datos de la ENIGH (ver Tabla A.6 en el Anexo) no es verificable con datos poblacionales para todos los años de la muestra.

${ }^{9}$ Se excluyen a aquellos jefes de familia que no se puede determinar su nivel educativo.
} 


\section{Tabla 1. Tamaño de Celda por Cohorte y Año de Levantamiento de la ENIGH}

2000-2016

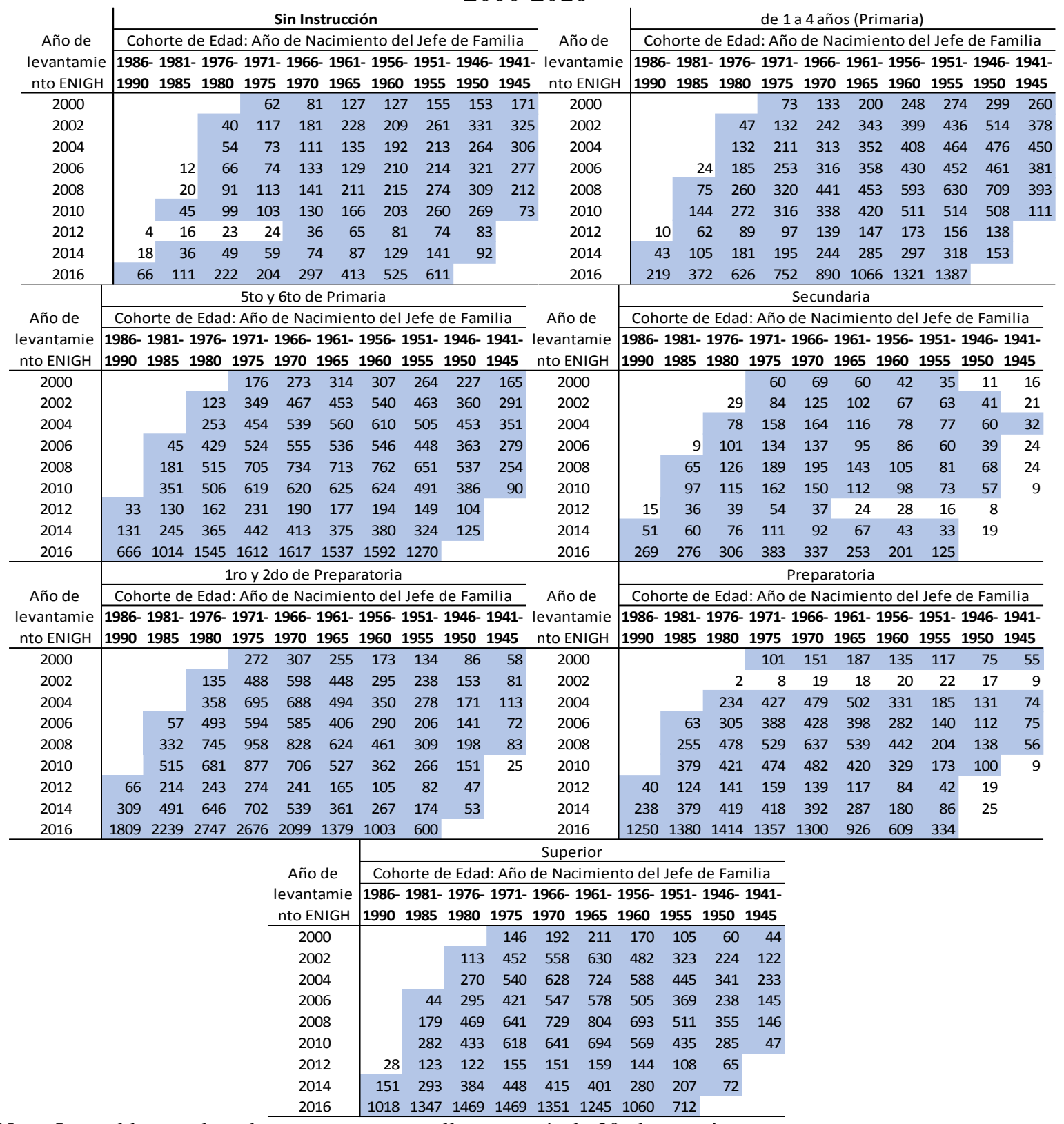

Nota: Las celdas sombreadas representan aquellas con más de 30 observaciones.

Fuente: Elaboración Propia con datos de INEGI-ENIGH 2000-2016.

Cabe señalar que se cuenta con un panel no balanceado, y que se utilizan para el análisis

solamente las celdas con más de 30 observaciones para evitar cualquier sesgo en los 
promedios de los ingresos y el consumo de las celdas al utilizar celdas con pocas observaciones. ${ }^{10}$

Dentro de las limitantes de utilizar el panel sintético, se debe señalar que estamos capturando los efectos entre las cohortes, y cualquier cambio en la varianza intra-cohorte no puede ser capturado por el modelo. Asimismo, y para ver si las cohortes del panel sintético corresponden en promedio a los datos del levantamiento, en el apéndice A6 se muestra la estadística descriptiva de las cohortes para el panel sintético y para los datos de la ENIGH con factores de expansión, utilizando las mismas definiciones de cohorte del panel sintético.

En la figura 2, se puede observar una primera aproximación de los cambios totales en el ingreso y el consumo para los distintos levantamientos de la ENIGH basándonos en el panel sintético. Dado que las varianzas del consumo y del ingreso presentan distintos niveles se utilizan dos escalas distintas en cada gráfica, en el eje izquierdo se miden los cambios en la varianza del ingreso en tanto que el eje derecho se presentan las del consumo. Los resultados indican que, en México para el periodo 2000-2016, dichas medidas de desigualdad siguen patrones muy similares a nivel nacional, lo que contrasta con el caso de Estados Unidos (Blundell et al., 2008) entre 1980-1992, donde se aprecia que la varianza del ingreso aumenta en mayor medida que la varianza del consumo a partir de la mitad de la década de los ochentas. Es preciso señalar que se la varianza del consumo aumenta en los levantamientos posteriores a recesiones, como la de 2001 (capturada en el levantamiento de 2002), ${ }^{11}$ se reduce substancialmente hacia el 2008, y vuelve a subir tras la crisis financiera de 2008 (capturada en el levantamiento de 2010 y 2012, al parecer menos intensa pero de mayor duración).

\footnotetext{
${ }^{10}$ Aunque las regresiones que se realizan más adelante están ponderadas por tamaño de cada una de las celdas, se quiso restringir aquellas celdas con menos de treinta hogares en su muestra.

${ }^{11}$ El periodo de recesión duró 36 meses, de octubre de 2000 a septiembre de 2003 (Heath, 2011).
} 
Figura 2. Comportamiento de la Varianza del Ingreso y el Consumo, para el panel sintético, Nacional y Regional, 2000-2016
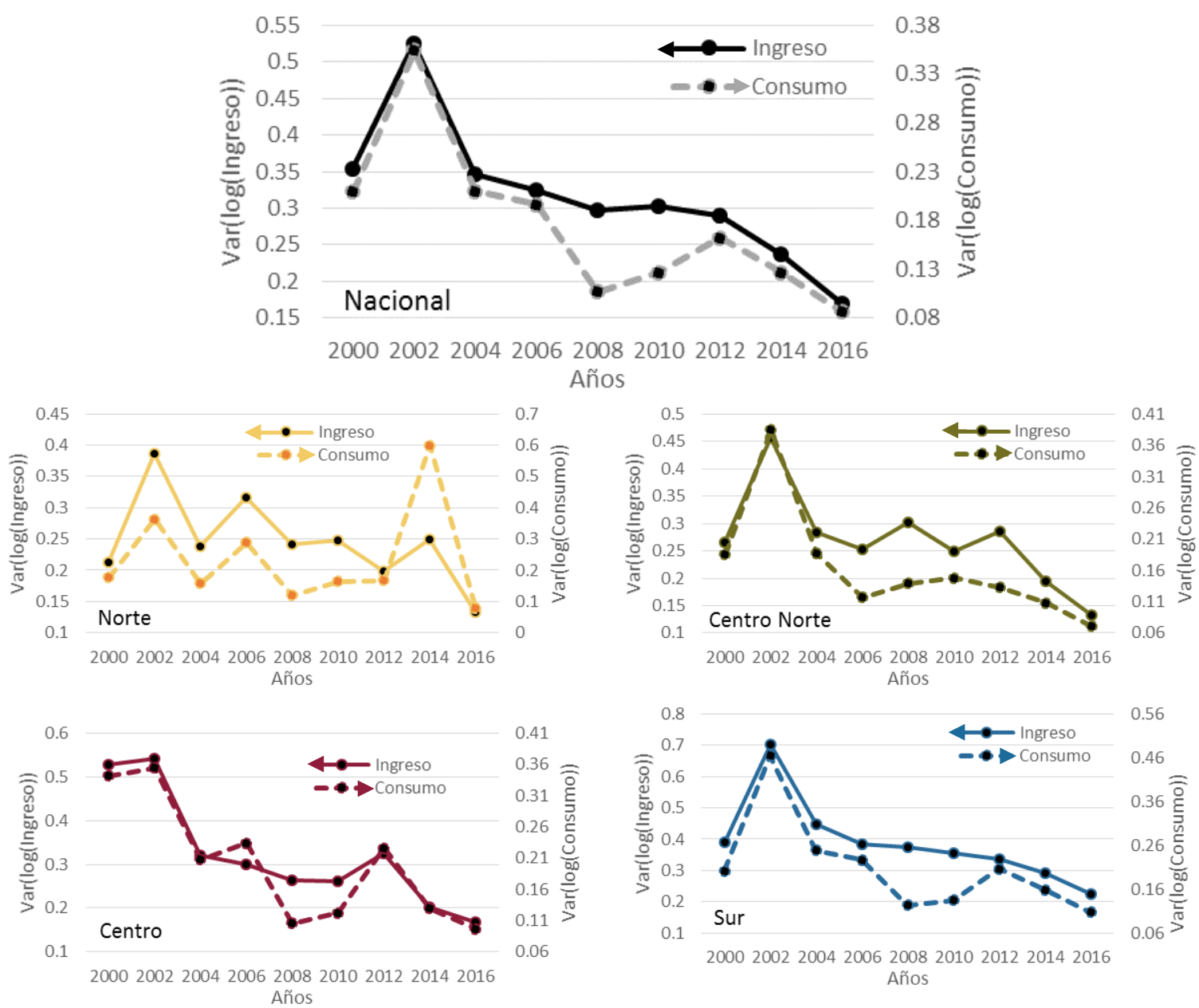

Fuente: Elaboración propia con datos del INEGI-ENIGH 2000-2016.

Cabe destacar que, al dividir la muestra a nivel regional, se exhibe cierto grado de heterogeneidad; siendo la región norte del país, la que muestra un comportamiento más distinto.

En relación a las características sociodemográficas que influyen en el ingreso y gasto de los hogares, se analizan en este documento de investigación el promedio del número de integrantes del hogar con menos de 10 años, para el periodo 2000-2016 en cada uno de los cohortes (tabla 2), el promedio por cohorte del número de perceptores por hogar (tabla 3), el número de horas trabajadas promedio por cohorte (tabla 4), la educación del cónyuge (tabla 
5), la proporción de hogares urbanos, y de hogares cuyo jefe de familia tiene acceso a la seguridad social $^{12}$ (tablas 6 y 7 ).

La tabla 2 presenta el número promedio de integrantes con menos de 10 años dentro de cada hogar promedio para el periodo completo de análisis. Como es de esperarse, el promedio de los integrantes del hogar con menos de 10 años en los cohortes más jóvenes y con menor instrucción es más elevado que en los cohortes con mayores niveles educativos y que cuentan con edades más avanzadas en relación al resto.

\section{Tabla 2. Número de Integrantes del Hogar con Menos de 10 años de Edad Promedio por Cohorte de Edad y Educativo, 2000-2016}

\begin{tabular}{|c|c|c|c|c|c|c|c|c|c|c|}
\hline \multirow{2}{*}{$\begin{array}{c}\text { Cohorte Educativo: } \\
\text { Educación del Jefe } \\
\text { de Familia }\end{array}$} & \multicolumn{10}{|c|}{ Cohorte de Edad: Año de Nacimiento del Jefe de Familia } \\
\hline & $\begin{array}{l}1986- \\
1990\end{array}$ & $\begin{array}{l}1981- \\
1985\end{array}$ & $\begin{array}{l}1976- \\
1980\end{array}$ & $\begin{array}{l}1971- \\
1975\end{array}$ & $\begin{array}{l}1966- \\
1970\end{array}$ & $\begin{array}{l}1961- \\
1965\end{array}$ & $\begin{array}{l}1956- \\
1960\end{array}$ & $\begin{array}{l}1951- \\
1955\end{array}$ & $\begin{array}{l}1946- \\
1950\end{array}$ & $\begin{array}{l}1941- \\
1945\end{array}$ \\
\hline Sin Instrucción & 2.17 & 2.08 & 2.15 & 1.84 & 1.60 & 1.25 & 1.14 & 0.96 & 0.85 & 0.69 \\
\hline Primaria 1-4 & 1.77 & 2.09 & 1.90 & 1.79 & 1.55 & 1.20 & 1.03 & 0.79 & 0.64 & 0.55 \\
\hline Primaria 5-6 & 1.82 & 1.80 & 1.79 & 1.68 & 1.36 & 1.04 & 0.78 & 0.63 & 0.55 & 0.47 \\
\hline Secundaria & 2.13 & 1.79 & 1.71 & 1.40 & 1.25 & 0.91 & 0.72 & 0.46 & 0.48 & 0.29 \\
\hline Preparatoria 1-2 & 1.73 & 1.66 & 1.55 & 1.42 & 1.18 & 0.87 & 0.60 & 0.55 & 0.48 & 0.43 \\
\hline Preparatoria & 1.41 & 1.38 & 1.20 & 1.10 & 1.02 & 0.77 & 0.54 & 0.42 & 0.34 & 0.22 \\
\hline Superior & 0.87 & 0.91 & 1.07 & 1.15 & 1.05 & 0.74 & 0.50 & 0.35 & 0.31 & 0.27 \\
\hline
\end{tabular}

\section{Menor número de integrantes menores a 10 años Mayor número de integrantes menores a 10 años}

Fuente: Elaboración propia con datos del INEGI-ENIGH 2000-2016.

Por su parte, la tabla 3 muestra el número promedio de perceptores del hogar en cada cohorte para el periodo 2000-2016. Se puede observar que el mayor número se concentra entre los grupos de edad 1 y 2 (aquellos nacidos entre 1981 y 1990) y en los grupos con grado de instrucción primaria o menor, disminuyendo gradualmente a mayor edad y educación del jefe de familia.

\footnotetext{
${ }^{12}$ La educación del conyugue está medida por años de escolaridad; la definición de hogar urbano está determinado por el tamaño de la localidad (más de 2 mil 500 habitantes); para la variable de acceso a la seguridad social, se determinó por el acceso a servicios de salud como prestación laboral (v.gr. IMSS).
} 
Tabla 3. Número de Perceptores por Hogar Promedio por Cohorte de Edad y Educativo, 2000-2016

Cohorte Educativo: Educación del Jefe de Familia

Sin Instrucción
Primaria 1-4
Primaria 5-6
Secundaria
Preparatoria 1-2
Preparatoria
Superior

\begin{tabular}{|c|c|c|c|c|c|c|c|c|c|}
\hline $\begin{array}{l}1986- \\
1990\end{array}$ & $\begin{array}{l}1981- \\
1985\end{array}$ & $\begin{array}{l}1976- \\
1980\end{array}$ & $\begin{array}{l}1971- \\
1975\end{array}$ & $\begin{array}{l}1966- \\
1970\end{array}$ & $\begin{array}{l}1961- \\
1965\end{array}$ & $\begin{array}{l}1956- \\
1960\end{array}$ & $\begin{array}{l}1951- \\
1955\end{array}$ & $\begin{array}{l}1946- \\
1950\end{array}$ & $\begin{array}{l}1941- \\
1945\end{array}$ \\
\hline 3.04 & 2.69 & 2.49 & 2.25 & 2.24 & 2.24 & 2.40 & 2.36 & 2.25 & 2.05 \\
\hline 2.47 & 2.70 & 2.29 & 2.24 & 2.28 & 2.31 & 2.31 & 2.21 & 2.08 & 1.97 \\
\hline 2.65 & 2.49 & 2.29 & 2.18 & 2.15 & 2.18 & 2.15 & 2.11 & 2.07 & 1.90 \\
\hline 2.80 & 2.50 & 2.25 & 2.11 & 2.18 & 2.04 & 2.01 & 2.00 & 1.87 & 1.61 \\
\hline 2.44 & 2.28 & 2.14 & 2.05 & 2.02 & 2.07 & 1.95 & 2.02 & 2.00 & 1.85 \\
\hline 2.08 & 2.03 & 1.89 & 1.93 & 1.94 & 1.89 & 1.88 & 1.98 & 1.79 & 1.61 \\
\hline \multirow[t]{3}{*}{1.35} & 1.53 & 1.70 & 1.83 & 1.88 & 1.85 & 1.84 & 1.79 & 1.79 & 1.65 \\
\hline & \multicolumn{9}{|c|}{ Menor número de perceptores } \\
\hline & \multicolumn{9}{|c|}{ Mayor número de perceptores } \\
\hline
\end{tabular}

Cohorte de Edad: Año de Nacimiento del Jefe de Familia

Mayor número de perceptores

Fuente: Elaboración propia con datos del INEGI-ENIGH 2000-2016.

El número de horas trabajadas por perceptor y por hogar, en cada uno de los cohortes promedio durante 2000 a 2016 se presenta en la tabla 4. Podemos destacar un alto número de horas trabajadas entre el cohorte con nivel de instrucción del jefe de familia con grado superior y jóvenes (primer grupo de edad) con 54.23 horas a la semana promedio, seguido por un segundo conjunto de cohortes que obtienen su máximo en el grupo de edad 7 (nacidos entre 1956 a 1960). 


\section{Tabla 4. Número de Horas Trabajadas por Perceptor y por Hogar Promedio por Cohorte de Edad y Educativo, 2000-2016}

\begin{tabular}{c}
$\begin{array}{c}\text { Cohorte Educativo: } \\
\text { Educación del Jefe } \\
\text { de Familia }\end{array}$ \\
\hline Sin Instrucción \\
Primaria 1-4 \\
Primaria 5-6 \\
Secundaria \\
Preparatoria 1-2 \\
Preparatoria \\
Superior
\end{tabular}

Cohorte de Edad: Año de Nacimiento del Jefe de Familia

\begin{tabular}{|c|c|c|c|c|c|c|c|c|c|}
\hline $\begin{array}{l}1986- \\
1990\end{array}$ & $\begin{array}{l}1981- \\
1985\end{array}$ & $\begin{array}{l}1976- \\
1980\end{array}$ & $\begin{array}{l}1971- \\
1975\end{array}$ & $\begin{array}{l}1966- \\
1970\end{array}$ & $\begin{array}{l}1961- \\
1965\end{array}$ & $\begin{array}{l}1956- \\
1960\end{array}$ & $\begin{array}{l}1951- \\
1955\end{array}$ & $\begin{array}{l}1946- \\
1950\end{array}$ & $\begin{array}{l}1941- \\
1945\end{array}$ \\
\hline 18.47 & 21.68 & 26.22 & 29.44 & 35.17 & 38.83 & 38.45 & 40.60 & 40.46 & 39.30 \\
\hline 25.89 & 22.60 & 29.08 & 32.56 & 36.27 & 40.73 & 42.80 & 43.78 & 42.79 & 41.89 \\
\hline 22.77 & 25.95 & 28.31 & 33.10 & 38.80 & 42.45 & 46.01 & 46.44 & 43.70 & 42.15 \\
\hline 22.57 & 27.72 & 28.83 & 35.19 & 37.82 & 46.35 & 47.95 & 46.97 & 43.72 & 42.72 \\
\hline 27.19 & 28.22 & 32.15 & 35.28 & 40.22 & 43.40 & 46.68 & 45.35 & 43.32 & 41.60 \\
\hline 35.62 & 34.77 & 33.93 & 36.07 & 39.29 & 42.74 & 44.41 & 40.77 & 42.72 & 42.68 \\
\hline 54.23 & 45.27 & 41.26 & 37.84 & 38.32 & 40.38 & 42.94 & 43.36 & 42.64 & 38.96 \\
\hline
\end{tabular}

\section{\begin{tabular}{|l|l} 
Menor número de horas trabajadas \\
\cline { 1 - 2 } Mayor número de horas trabajadas
\end{tabular}}

Fuente: Elaboración propia con datos del INEGI-ENIGH 2000-2016.

En la tabla 5 se presenta la educación del cónyuge en cada uno de los cohortes promedio durante 2000 a 2016. Aquí se destaca la relación lineal que tiene con la edad (cónyuge en cohortes más jóvenes tienden a tener mayor educación) y con la educación del jefe(a) de familia (a mayor educación del jefe(a) de familia, mayor educación de la pareja).

Tabla 5. Años de Educación del Cónyuge

\section{Promedio por Cohorte de Edad y Educativo, 2000-2016}

\begin{tabular}{|c|c|c|c|c|c|c|c|c|c|c|}
\hline \multirow{2}{*}{$\begin{array}{c}\text { Cohorte Educativo: } \\
\text { Educación del Jefe } \\
\text { de Familia }\end{array}$} & \multicolumn{10}{|c|}{ Cohorte de Edad: Año de Nacimiento del Jefe de Familia } \\
\hline & $\begin{array}{l}1986- \\
1990\end{array}$ & $\begin{array}{l}1981- \\
1985\end{array}$ & $\begin{array}{l}1976- \\
1980\end{array}$ & $\begin{array}{l}1971- \\
1975\end{array}$ & $\begin{array}{l}1966- \\
1970\end{array}$ & $\begin{array}{l}1961- \\
1965\end{array}$ & $\begin{array}{l}1956- \\
1960\end{array}$ & $\begin{array}{l}1951- \\
1955\end{array}$ & $\begin{array}{l}1946- \\
1950\end{array}$ & $\begin{array}{l}1941- \\
1945\end{array}$ \\
\hline Sin Instrucción & 5.88 & 3.60 & 3.11 & 3.10 & 2.77 & 2.46 & 1.77 & 1.54 & 1.13 & 0.85 \\
\hline Primaria 1-4 & 6.20 & 4.73 & 4.54 & 4.01 & 3.86 & 3.46 & 2.94 & 2.66 & 2.40 & 1.98 \\
\hline Primaria 5-6 & 6.94 & 6.00 & 5.66 & 5.35 & 5.03 & 4.65 & 4.29 & 3.99 & 3.56 & 3.38 \\
\hline Secundaria & 6.56 & 7.10 & 6.45 & 6.65 & 6.27 & 5.85 & 5.59 & 5.30 & 4.43 & 4.81 \\
\hline Preparatoria 1-2 & 7.62 & 7.55 & 7.45 & 7.25 & 6.92 & 6.55 & 6.07 & 5.64 & 5.20 & 5.07 \\
\hline Preparatoria & 8.91 & 8.85 & 7.81 & 8.69 & 9.87 & 9.78 & 9.00 & 8.92 & 9.32 & 7.49 \\
\hline Superior & 9.94 & 9.57 & 10.73 & 11.08 & 10.49 & 10.17 & 9.80 & 9.90 & 9.02 & 8.97 \\
\hline
\end{tabular}

Menor número de escolaridad en años

Mayor número de escolaridad en años

Fuente: Elaboración propia con datos del INEGI-ENIGH 2000-2016. 
Para la proporción de hogares promedio que vive en localidades urbanas de acuerdo con la ENIGH (tabla 6), se puede observar una clara división por el nivel de educación del jefe(a) de familia, concentrándose en localidades urbanas los cohortes con mayor educación y mayor edad.

Tabla 6. Proporción de Hogares Urbanos por Cohorte de Edad y Educativo, 2000-2016

\begin{tabular}{|c|c|c|c|c|c|c|c|c|c|c|}
\hline \multirow{2}{*}{$\begin{array}{c}\text { Cohorte Educativo: } \\
\text { Educación del Jefe } \\
\text { de Familia }\end{array}$} & \multicolumn{10}{|c|}{ Cohorte de Edad: Año de Nacimiento del Jefe de Familia } \\
\hline & $\begin{array}{l}1986- \\
1990\end{array}$ & $\begin{array}{l}1981- \\
1985\end{array}$ & $\begin{array}{l}1976- \\
1980\end{array}$ & $\begin{array}{l}1971- \\
1975\end{array}$ & $\begin{array}{l}1966- \\
1970\end{array}$ & $\begin{array}{l}1961- \\
1965\end{array}$ & $\begin{array}{l}1956- \\
1960\end{array}$ & $\begin{array}{l}1951- \\
1955\end{array}$ & $\begin{array}{l}1946- \\
1950\end{array}$ & $\begin{array}{l}1941- \\
1945\end{array}$ \\
\hline Sin Instrucción & $53 \%$ & $39 \%$ & $43 \%$ & $43 \%$ & $46 \%$ & $47 \%$ & $48 \%$ & $47 \%$ & $49 \%$ & $51 \%$ \\
\hline Primaria 1-4 & $45 \%$ & $48 \%$ & $45 \%$ & $45 \%$ & $49 \%$ & $51 \%$ & $52 \%$ & $54 \%$ & $58 \%$ & $62 \%$ \\
\hline Primaria 5-6 & $44 \%$ & $50 \%$ & $53 \%$ & $56 \%$ & $59 \%$ & $65 \%$ & $72 \%$ & $75 \%$ & $81 \%$ & $83 \%$ \\
\hline Secundaria & $61 \%$ & $69 \%$ & $71 \%$ & $78 \%$ & $74 \%$ & $83 \%$ & $83 \%$ & $86 \%$ & $86 \%$ & $93 \%$ \\
\hline Preparatoria 1-2 & $57 \%$ & $67 \%$ & $73 \%$ & $75 \%$ & $77 \%$ & $81 \%$ & $83 \%$ & $85 \%$ & $91 \%$ & $90 \%$ \\
\hline Preparatoria & $75 \%$ & $82 \%$ & $86 \%$ & $89 \%$ & $88 \%$ & $91 \%$ & $91 \%$ & $92 \%$ & $95 \%$ & $94 \%$ \\
\hline Superior & $89 \%$ & $91 \%$ & $92 \%$ & $92 \%$ & $92 \%$ & $93 \%$ & $94 \%$ & $95 \%$ & $95 \%$ & $95 \%$ \\
\hline
\end{tabular}

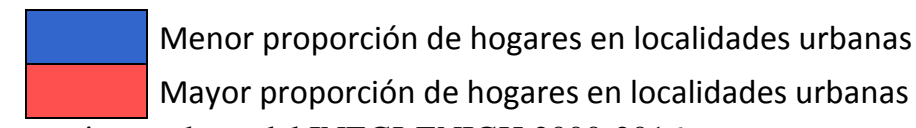

Fuente: Elaboración propia con datos del INEGI-ENIGH 2000-2016.

Para el acceso a los servicios de seguridad social para el jefe de familia (tabla 7), se puede observar una clara concentración en los cohortes con mayor nivel educativo y jóvenes, con porcentajes superiores al 60\% para los que ostentan algún grado de educación universitaria. En contraste, los grados de informalidad están concentrados en cohortes con mayor edad y menor educación. 


\section{Tabla 7. Hogares cuyo Jefe de Familia Tiene Acceso a la Seguridad Social Proporción Promedio por Cohorte de Edad y Educativo, 2000-2016}

\begin{tabular}{l}
$\begin{array}{c}\text { Cohorte Educativo } \\
\text { Educación del Jefe } \\
\text { de Familia }\end{array}$ \\
\hline Sin Instrucción \\
Primaria 1-4 \\
Primaria 5-6 \\
Secundaria \\
Preparatoria 1-2 \\
Preparatoria \\
Superior
\end{tabular}

\begin{tabular}{|c|c|c|c|c|c|c|c|c|c|}
\hline $\begin{array}{l}1986- \\
1990\end{array}$ & $\begin{array}{l}1981- \\
1985\end{array}$ & $\begin{array}{l}1976- \\
1980\end{array}$ & $\begin{array}{l}1971- \\
1975\end{array}$ & $\begin{array}{l}1966- \\
1970\end{array}$ & $\begin{array}{l}1961- \\
1965\end{array}$ & $\begin{array}{l}1956- \\
1960\end{array}$ & $\begin{array}{l}1951- \\
1955\end{array}$ & $\begin{array}{l}1946- \\
1950\end{array}$ & $\begin{array}{l}1941- \\
1945\end{array}$ \\
\hline $7 \%$ & $6 \%$ & $8 \%$ & $7 \%$ & $7 \%$ & $7 \%$ & $6 \%$ & $7 \%$ & $5 \%$ & $5 \%$ \\
\hline $9 \%$ & $13 \%$ & $11 \%$ & $13 \%$ & $13 \%$ & $13 \%$ & $12 \%$ & $12 \%$ & $10 \%$ & $9 \%$ \\
\hline $20 \%$ & $19 \%$ & $21 \%$ & $21 \%$ & $21 \%$ & $23 \%$ & $22 \%$ & $20 \%$ & $17 \%$ & $15 \%$ \\
\hline $23 \%$ & $22 \%$ & $29 \%$ & $33 \%$ & $30 \%$ & $30 \%$ & $29 \%$ & $23 \%$ & $18 \%$ & $14 \%$ \\
\hline $29 \%$ & $37 \%$ & $41 \%$ & $40 \%$ & $39 \%$ & $39 \%$ & $37 \%$ & $32 \%$ & $25 \%$ & $23 \%$ \\
\hline $51 \%$ & $49 \%$ & $50 \%$ & $51 \%$ & $53 \%$ & $53 \%$ & $49 \%$ & $40 \%$ & $37 \%$ & $29 \%$ \\
\hline $61 \%$ & $62 \%$ & $61 \%$ & $59 \%$ & $61 \%$ & $57 \%$ & $51 \%$ & $42 \%$ & $35 \%$ & $26 \%$ \\
\hline
\end{tabular}

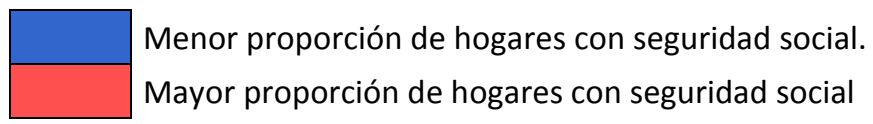

Fuente: Elaboración propia con datos del INEGI-ENIGH 2000-2016.

\section{Metodología}

Siguiendo el modelo presentado por Blundell et al. (2008), el cual tomamos para esta aplicación al caso mexicano, y que a su vez está basado en el modelo propuesto por Hall y Mishkin (1982) se descompone el ingreso en dos componentes: una parte idiosincrática, dependiente de las características demográficas de los hogares, y otra que corresponde al ingreso permanente.

$$
\ln Y_{i, t}=Z_{i, t}^{\prime} \varphi_{t}+P_{i, t}+v_{i, t}
$$

Donde $t$ es tiempo (o la indexación del levantamiento de la encuesta ENIGH, que se encuentra entre 2000 y 2016, por lo que $t \in[1,9]$ ), $i$ es el hogar (o cohorte en un panel sintético), $\boldsymbol{Z}$ es una matriz de variables demográficas que influyen en el ingreso ${ }^{13}$ y $P$ denota ingreso permanente. Dicho ingreso permanente sigue un proceso de caminata aleatoria expresado de la siguiente forma:

\footnotetext{
${ }^{13}$ Dichas variables son: Número de menores de 10 años en el hogar, Cantidad de Perceptores por hogar, Si el hogar se encuentra en una localidad urbana o rural, educación formal del esposo(a), si el jefe de familia tiene acceso a seguridad social, y efectos fijos por cohorte y año de levantamiento.
} 


$$
P_{i, t}=P_{i, t-1}+\zeta_{i, t}
$$

Donde $\zeta_{i, t}$ corresponde a los choques permanentes al ingreso (el coeficiente del rezago del ingreso permanente se supone igual a uno). Es decir, un choque en un momento arbitrario $t_{1}$, donde $t_{1} \in[1,9]$; modificaría definitivamente el nivel del ingreso permanente a partir de dicho punto. Se asume también que los choques permanentes al ingreso $\zeta_{i, t}$, no están correlacionados serialmente.

Adicionalmente, se asume que el componente transitorio $v_{i, t}$ del ingreso sigue un proceso de media móvil $M A(q)$

$$
v_{i, t}=\sum_{j=0}^{q} \theta_{j} \varepsilon_{i, t-j}
$$

Por lo tanto, si se define el logaritmo del ingreso no explicado $\left(y_{i, t}\right)$ como aquel neto de sus efectos sociodemográficos $y_{i, t}=\ln Y_{i, t}-\boldsymbol{Z}_{i, t}^{\prime} \varphi_{t}$, tendríamos que su crecimiento estaría explicado por sus choques permanentes y los transitorios:

$$
\Delta y_{i, t}=\zeta_{i, t}+\Delta v_{i, t}
$$

Cabe destacar que de acuerdo con Hall y Mishkin (1982), una característica clave del modelo es la hipótesis de que los hogares conocen, de manera separada, los dos componentes estocásticos del ingreso.

Por su parte, el cambio en el logaritmo del consumo se asume se comporta de la siguiente forma (Jappelli y Pistaferri, 2017):

$$
\Delta \ln C_{i t}=Z_{i, t}^{\prime} \lambda+\alpha E_{t-1} \Delta \ln Y_{i t}+\phi_{i, t} \zeta_{i, t}+\psi_{i, t} \varepsilon_{i, t}+\xi_{i, t}
$$

Donde $Z_{i, t}$ son las características sociodemográficas que afectan al consumo, $E_{t-1} \Delta \ln Y_{i t}$ son los cambios esperados en el ingreso (que asumiremos no afectan a los cambios en el 
consumo $\left.^{14}\right), \zeta_{i, t}$ es el choque al ingreso permanente, $\varepsilon_{i, t}$ representa el choque transitorio, $\mathrm{y}$ $\xi_{i, t}$ es el error de aproximación (que puede incluir el error de medición).

De la misma forma que el ingreso, definiendo $c_{i, t}$ como el logaritmo del consumo que no es explicado por sus características sociodemográficas $\left(\Delta c_{i, t}=\Delta \ln C_{i, t}-Z_{i, t}^{\prime} \varphi_{t}^{c}\right)$ y asumiendo que $\alpha=0$, la transmisión de los choques no anticipados del ingreso al consumo estaría determinada por la siguiente relación:

$$
\Delta c_{i, t}=\phi_{i, t} \zeta_{i, t}+\psi_{i, t} \varepsilon_{i, t}+\xi_{i, t}
$$

De tal forma que el choque permanente al ingreso se relaciona al consumo vía $\phi$, el cual puede variar por tiempo e individuo o cohorte; mientras que el choque transitorio al ingreso se relaciona por medio de $\psi$; y las innovaciones en el consumo independientes del ingreso estarán medidos por $\xi_{i, t}$.

Así, el modelo permite probar distintas hipótesis. i) ingreso permanente, la cual establece que choques en el ingreso permanente impactarán el consumo $\left(\phi_{i, t}=1\right)$, mientras que los choques temporales no tendrán efecto alguno $\left(\psi_{i, t}=0\right)$, ya que las familias podrán suavizar el consumo ante cambios temporales en el ingreso; ii) mercados completos (aseguramiento completo de choques en el ingreso) sería el caso donde $\phi_{i, t}=\psi_{i, t}=0$, y mercados $\sin$ aseguramiento en los choques (permanentes o temporales) en el ingreso que podría probarse mediante la restricción $\phi_{i, t}=\psi_{i, t}=1$; iii) ahorro precautorio (o aseguramiento parcial), que permite a las familias asegurar los choques permanentes mediante el ahorro, lo cual permite parámetros menores que la teoría del ingreso permanente, por lo que $0<\phi_{i, t}<1$, con un impacto moderado o nulo para los choques temporales $\left(\psi_{i, t}\right)$.

Cabe destacar que no es posible estimar la ecuación 4 de manera directa debido a que el modelo está subidentificado: $\psi_{i, t}$ no es conocida; la varianza del choque transitorio $\operatorname{Var}\left(\varepsilon_{t}\right)$ y el error de medición en el ingreso $\operatorname{Var}\left(\Delta v_{t}\right)$ no pueden ser identificados de manera separada (Jappelli y Pistaferri, 2017). Por lo anterior, la identificación del modelo con datos

\footnotetext{
${ }^{14}$ En efecto, "modelos con mercados crediticios perfectos y utilidad cuadrática implican que el consumo no debería variar con cambios anticipados en el ingreso" (Jappelli y Pistaferri, 2017)
} 
panel de ingreso y consumo, basados en las ecuaciones 3 y 4 debe ser aproximada con una serie de restricciones de las varianzas del crecimiento neto de los ingresos y los consumos y sus respectivas covarianzas.

Las restricciones de varianzas y covarianzas ${ }^{15}$ son:

$$
\begin{aligned}
& \operatorname{Var}\left(\Delta y_{t}\right)=\operatorname{Var}\left(\zeta_{t}\right)+\operatorname{Var}\left(\Delta v_{t}\right) \\
& \operatorname{Cov}\left(\Delta y_{t}, \Delta y_{t+s}\right)=\operatorname{Cov}\left(\Delta v_{t}, \Delta v_{t+s}\right) \\
& \operatorname{Var}\left(\Delta c_{t}\right)=\phi^{2} \operatorname{Var}\left(\zeta_{t}\right)+\psi^{2} \operatorname{Var}\left(\varepsilon_{t}\right)+\operatorname{Var}\left(\xi_{t}\right) \\
& \operatorname{Cov}\left(\Delta c_{t}, \Delta y_{t}\right)=\phi_{t} \operatorname{Var}\left(\zeta_{t}\right)+\psi_{t} \operatorname{Var}\left(\varepsilon_{t}\right) \\
& \operatorname{Cov}\left(\Delta c_{t}, \Delta y_{t+s}\right)=\psi_{t} \operatorname{Cov}\left(\varepsilon_{t}, \Delta v_{t+s}\right)
\end{aligned}
$$

Donde $t$ denota año, y $s$ son incrementos anuales tales que $s \in[1, T-t]$; la varianza del ingreso (5) y su covarianza intertemporal (6) provienen de la ecuación del ingreso (3); la varianza del consumo (7) y las covarianzas del consumo e ingreso ( 8 y 9) provienen de la ecuación (4), en donde la covarianza intertemporal del consumo debe ser cero, debido a que se asume que el consumo sigue un proceso de martingala.

Cabe destacar que para la ecuación 6, la covarianza depende de las propiedades de media móvil del componente transitorio del ingreso $v_{i, t}$. Siendo así, si el proceso es un MA(q), cualquier covarianza donde $s>\mathrm{q}$, debe ser igual a cero, es decir, para un MA(1), una autocovarianza en el ingreso entre $\Delta y_{t}, \Delta y_{t+3}$ debe ser no significativa.

Como primer paso en la estimación, se tomarán los residuales de la regresión entre el logaritmo del ingreso y sus variables sociodemográficas, la cual sería $\hat{y}_{i, t}$. Lo mismo con la ecuación de consumo, para la construcción de $\hat{c}_{i, t}$. A dichos estimadores se estimarán sus varianzas y covarianzas por mínima distancia (básicamente utilizando mínimos cuadrados ordinarios para cada año) para conocer su composición. Así, una covarianza significativa entre el ingreso y el consumo implicaría, de acuerdo con la ecuación (8) $\phi_{t} \operatorname{Var}\left(\zeta_{t}\right)+$

\footnotetext{
${ }^{15}$ En la explicación de dichas restricciones, Japelli y Pistaferry (2017) utilizan otra notación. En este documento utilizamos el listado de Japelli y Pistaferri (2017) con la notación de Blundell et al. (2008).
} 
$\psi_{t} \operatorname{Var}\left(\varepsilon_{t}\right)>0$, y una covarianza no significativa de orden uno, de acuerdo con la ecuación (9) de $\psi_{t} \operatorname{Cov}\left(\varepsilon_{t}, \Delta v_{t+s}\right)=0$, por lo que se podría inferir la posibilidad de que exista evidencia de un choque permanente, y que el choque transitorio no sea distinto estadísticamente de cero.

Si bien dichas estimaciones nos pueden dar una noción del sentido de dichos coeficientes, para poder identificarlos propiamente, se debe resolver el sistema mediante mínima distancia diagonalmente ponderada. En esta investigación seguimos el procedimiento descrito por Blundell et al. (2008) en su Apéndice C. ${ }^{16}$ En tanto que la inferencia (matriz de varianzas y covarianzas) se obtiene por el Jacobiano de la minimización numérica de DWMD.

\section{Resultados}

Debido a que es necesario trabajar con la parte no determinística del ingreso y el consumo, se remueven los impactos de las características sociodemográficas de las distintas cohortes estimando los errores de la regresión de los ingresos y el consumo de no durables con respecto a las características sociodemográficas y variables dicotómicas de cohorte (año de nacimiento y educación del jefe de familia) ${ }^{17}$, estas nuevas variables (los errores respectivos a la ecuación de ingreso y de consumo) capturan los cambios no explicados por los elementos determinísticos.

Con esta variable (que se denomina en la ecuación 3 y $4 c_{i, t}$ y $y_{i, t}$ para el consumo y el ingreso, respectivamente) se estiman las matrices de autocovarianza del ingreso y del consumo a nivel nacional y regional para diferentes rezagos (tabla 8). Este paso es necesario para determinar la naturaleza del comportamiento del ingreso y el consumo en el tiempo. Se pueden observar dos puntos máximos en la varianza del ingreso, el primero en el año 2004 y el segundo en 2014, para luego descender para el levantamiento de la ENIGH del 2016. La autocovarianza del ingreso es significativa para los años 2006 y 2012, aunado a que las

\footnotetext{
${ }^{16} \mathrm{Si}$ bien los autores originalmente escribieron la rutina en GAUSS, se logró replicar sus resultados con el comando "optim" en R.

${ }^{17}$ Las regresiones se ponderaron de acuerdo al tamaño de cada celda del panel sintético. Los resultados de las regresiones se muestran en la tabla A5, en los Anexos.
} 
autocovarianzas de mayor grado (un rezago o más) pierden significancia estadística, siendo sólo estadísticamente distinto de cero el año 2008 y 2012.

En el caso de la matriz de autocovarianzas en el crecimiento del consumo no explicado, se puede observar un aumento hacia el 2004, y últimamente hacia el 2014. De la misma forma que el ingreso a nivel nacional por cohorte, la autocorrelación del consumo con su primer rezago es estadísticamente distinta de cero para 2002, 2006, 2010, 2012 y 2014, mientras que los rezagos de orden dos o mayor no muestran ser significativos con excepción del año 2004, 2008 y 2010. ${ }^{18}$ Es interesante destacar que las caídas en la varianza del consumo capturadas en la figura 2 se muestran con autocovarianzas negativas y significativas para los tres levantamientos posteriores al 2008. ${ }^{19}$

\footnotetext{
${ }^{18}$ En el anexo se muestran las matrices de autocovarianzas para cada una de las regiones.

${ }^{19}$ Una hipótesis podría ser el cambio metodológico de la ENIGH 2008, pero los coeficientes parten de los residuales de la regresión del consumo (el consumo no explicado) los cuales incluyen variables dicotómicas por cohorte y año de levantamiento (Tabla A5, en los Anexos). Una causa plausible pudiera ser los efectos de la crisis de 2008, los cuales pudieron ser de una duración mayor.
} 


\section{Tabla 8. Matrices de Covarianzas para el Crecimiento del Ingreso y el Consumo no explicado (ecuaciones 3 y 4), Nivel Nacional}

Matriz de Autocovarianzas del Crecimiento del Ingreso

\begin{tabular}{|c|c|c|c|}
\hline Año & $\operatorname{Var}\left(\Delta y_{t}\right)$ & $\operatorname{Cov}\left(\Delta y_{t+1}, \Delta y_{t}\right)$ & $\operatorname{Cov}\left(\Delta y_{t+2}, \Delta y_{t}\right)$ \\
\hline \multirow[t]{2}{*}{2002} & $0.012 * * *$ & 0.002 & -0.000 \\
\hline & $(0.002)$ & $(0.003)$ & $(0.001)$ \\
\hline \multirow[t]{2}{*}{2004} & $0.031 * * *$ & -0.003 & -0.000 \\
\hline & $(0.008)$ & $(0.002)$ & $(0.002)$ \\
\hline \multirow[t]{2}{*}{2006} & $0.005^{* * *}$ & $-0.003^{* * *}$ & 0.001 \\
\hline & $(0.002)$ & $(0.001)$ & $(0.001)$ \\
\hline \multirow[t]{2}{*}{2008} & $0.005^{* * *}$ & -0.001 & $-0.002 *$ \\
\hline & (0.001) & $(0.001)$ & $(0.001)$ \\
\hline \multirow[t]{2}{*}{2010} & $0.004^{* * *}$ & $-0.001 *$ & -0.000 \\
\hline & $(0.001)$ & $(0.001)$ & $(0.001)$ \\
\hline \multirow[t]{2}{*}{2012} & $0.008 * * *$ & $-0.005^{* * *}$ & $0.002 * *$ \\
\hline & $(0.002)$ & $(0.002)$ & $(0.001)$ \\
\hline \multirow[t]{2}{*}{2014} & $0.010 * * *$ & -0.000 & \\
\hline & $(0.002)$ & $(0.001)$ & \\
\hline \multirow[t]{2}{*}{2016} & $0.008^{* * *}$ & & \\
\hline & $(0.002)$ & & \\
\hline \multicolumn{4}{|c|}{ Matriz de Autocovarianzas del Crecimiento del Consumo } \\
\hline Año & $\operatorname{Var}\left(\Delta c_{t}\right)$ & $\operatorname{Cov}\left(\Delta c_{t+1}, \Delta c_{t}\right)$ & $\operatorname{Cov}\left(\Delta c_{t+2}, \Delta c_{t}\right)$ \\
\hline \multirow[t]{2}{*}{2002} & $0.011^{* * *}$ & 0.002 & -0.001 \\
\hline & $(0.003)$ & $(0.002)$ & $(0.001)$ \\
\hline \multirow[t]{2}{*}{2004} & $0.022 * * *$ & -0.001 & $-0.004 *$ \\
\hline & $(0.006)$ & $(0.002)$ & $(0.002)$ \\
\hline \multirow[t]{2}{*}{2006} & $0.008 * * *$ & $-0.004 * * *$ & -0.001 \\
\hline & $(0.002)$ & $(0.001)$ & $(0.001)$ \\
\hline \multirow[t]{2}{*}{2008} & $0.012 * * *$ & -0.001 & -0.002 \\
\hline & $(0.002)$ & $(0.001)$ & $(0.001)$ \\
\hline \multirow[t]{2}{*}{2010} & $0.006^{* * *}$ & $-0.003 * *$ & $-0.002 * *$ \\
\hline & $(0.001)$ & $(0.001)$ & $(0.001)$ \\
\hline \multirow[t]{2}{*}{2012} & $0.006^{* * *}$ & $-0.003^{* * *}$ & -0.001 \\
\hline & $(0.001)$ & $(0.001)$ & $(0.001)$ \\
\hline \multirow[t]{2}{*}{2014} & $0.010 * * *$ & 0.002 & \\
\hline & $(0.002)$ & $(0.001)$ & \\
\hline \multirow[t]{2}{*}{2016} & $0.012^{* * *}$ & & \\
\hline & $(0.002)$ & & \\
\hline
\end{tabular}

Nota: Errores estándar en paréntesis. Significancia: *** al 1\%; ** al 5\%; * al 10\%.

Estimación de la Matriz de Autocovarianzas de la Ecuación 3 y 4. Las variables $\Delta c_{t}$ y $\Delta y_{t}$ corresponden al incremento del consumo e ingreso no explicado por variables sociodemográficas, respectivamente.

Fuente: Estimaciones propias con datos del INEGI.

En la tabla 9, se muestra la asociación del crecimiento del ingreso no explicado y el crecimiento del consumo. La covarianza entre el crecimiento no explicado del consumo y el ingreso en tiempo t es estadísticamente significativa, lo cual implica de acuerdo con la ecuación 8 que $\phi \operatorname{Var}\left(\zeta_{t}\right)+\psi \operatorname{Var}\left(\Delta v_{t}\right)>0$. Esta relación alcanzó un máximo en 2004 para 
luego descender al 2012 y ha mostrado recuperación hacia el año 2016. Asimismo, se observa que la covarianza del crecimiento del consumo no explicado y el crecimiento del ingreso no explicado a un periodo futuro es significativa para los años 2002, 2006 y 2010. Esta covarianza, de acuerdo con Blundell et al. (2008) representa el aseguramiento que las familias o cohortes muestran ante choques transitorios, en otras palabras, una covarianza igual a cero entre el crecimiento del ingreso no explicado futuro (a un periodo) y el crecimiento del consumo no explicado implica que las familias cubren dichos choques temporales.

\section{Tabla 9. Matrices de Covarianzas para el Crecimiento del Consumo y el Ingreso no} explicado (ecuaciones 3 y 4), Nivel Nacional

Matriz de Autocovarianzas del Crecimiento del Consumo-Ingreso

\begin{tabular}{|c|c|c|c|}
\hline Año & $\operatorname{Cov}\left(\Delta y_{t}, \Delta c_{t}\right)$ & $\operatorname{Cov}\left(\Delta y_{t+1}, \Delta c_{t}\right)$ & $\operatorname{Cov}\left(\Delta y_{t}, \Delta c_{t+1}\right)$ \\
\hline \multirow[t]{2}{*}{2002} & $0.065 * *$ & -0.036 & -0.045 \\
\hline & $(0.027)$ & $(0.031)$ & $(0.027)$ \\
\hline \multirow[t]{2}{*}{2004} & $0.109 * * *$ & -0.019 & -0.010 \\
\hline & $(0.038)$ & $(0.014)$ & $(0.011)$ \\
\hline \multirow[t]{2}{*}{2006} & $0.022 * *$ & -0.007 & -0.005 \\
\hline & $(0.011)$ & $(0.007)$ & $(0.006)$ \\
\hline \multirow[t]{2}{*}{2008} & 0.039 & 0.001 & -0.010 \\
\hline & $(0.025)$ & $(0.008)$ & $(0.014)$ \\
\hline \multirow[t]{2}{*}{2010} & $0.037^{* *}$ & -0.009 & -0.011 \\
\hline & $(0.015)$ & $(0.011)$ & $(0.007)$ \\
\hline \multirow[t]{2}{*}{2012} & $0.014 * *$ & -0.008 & $-0.016 *$ \\
\hline & $(0.007)$ & (0.010) & $(0.008)$ \\
\hline \multirow[t]{2}{*}{2014} & $0.037^{* * *}$ & $-0.048^{*}$ & $-0.036 * * *$ \\
\hline & $(0.012)$ & $(0.027)$ & $(0.013)$ \\
\hline \multirow[t]{2}{*}{2016} & $0.079 * *$ & & \\
\hline & $(0.038)$ & & \\
\hline \multicolumn{3}{|c|}{ Test $\operatorname{Cov}\left(\Delta \mathrm{y}_{\mathrm{t}+1}, \Delta \mathrm{c}_{\mathrm{t}}\right)=0$ para toda $\mathrm{t}$} & $p$-value $<0.1 \%$ \\
\hline \multicolumn{3}{|c|}{$\operatorname{Test} \operatorname{Cov}\left(\Delta \mathrm{y}_{\mathrm{t}+2}, \Delta \mathrm{c}_{\mathrm{t}}\right)=0$ para toda $\mathrm{t}$} & p-value 39\% \\
\hline
\end{tabular}

Nota: Errores estándar en paréntesis. Significancia: *** al 1\%; ** al 5\%; * al $10 \%$.

Estimación de la matriz de autocovarianzas basado en la ecuaciones 3 y 4 . Las variables $\Delta c_{t}$ y $\Delta y_{t}$ corresponden al incremento del consumo e ingreso no explicado por variables sociodemográficas, respectivamente.

Fuente: Estimación propia con datos del INEGI-ENIGH 2000-2016.

Combinando los resultados de las ecuaciones 8 y 9 tenemos que $\phi \operatorname{Var}\left(\zeta_{t}\right)+\psi \operatorname{Var}\left(\Delta v_{t}\right)>0$ y $\psi \operatorname{Cov}\left(\varepsilon_{t}, \Delta v_{t+s}\right)=0$. Lo anterior sugiere que hay indicios de que choques inesperados en el ingreso permanente pueden incidir en el crecimiento del consumo, y que no hay evidencia clara de que existan cambios en el consumo no explicado ante choques temporales en el 
ingreso ${ }^{20}$ sin embargo, es necesario estimar ambos coeficientes para tener un resultado más preciso.

En la tercera columna de la tabla 9, se muestran las covarianzas entre el crecimiento del consumo no explicado e incrementos pasados en el ingreso no explicado. Con excepción del año 2012 y 2014, los coeficientes no muestran ser estadísticamente diferentes de cero.

En lo que se refiere al análisis regional, para la región norte podemos observar que la varianza en el crecimiento tanto del ingreso como del consumo se ha mantenido constante (Ver Tabla A1, en el anexo). Para el análisis de los componentes temporal y permanente, se encuentran resultados similares a los del nivel nacional, aunque en este caso la evidencia es más débil, debido a que un año no es significativo en 2014 en la covarianza contemporánea del ingreso y consumo (primera columna de la tabla 10).

\section{Tabla 10. Matrices de Covarianzas para el Crecimiento del Consumo y el Ingreso no explicado (ecuaciones 3 y 4), Región Norte}

Matriz de Autocovarianzas del Crecimiento del Consumo-Ingreso

\begin{tabular}{rlll}
\hline Año & $\operatorname{Cov}\left(\Delta \mathrm{y}_{\mathrm{t}}, \Delta \mathrm{c}_{\mathrm{t}}\right)$ & $\operatorname{Cov}\left(\Delta \mathrm{y}_{\mathrm{t}+1}, \Delta \mathrm{c}_{\mathrm{t}}\right)$ & $\operatorname{Cov}\left(\Delta \mathrm{y}_{\mathrm{t}}, \Delta \mathrm{c}_{\mathrm{t}+1}\right)$ \\
\hline 2002 & $0.015^{* *}$ & -0.002 & 0.006 \\
& $(0.006)$ & $(0.003)$ & $(0.006)$ \\
2004 & $0.017^{* * *}$ & 0.002 & 0.006 \\
& $(0.004)$ & $(0.004)$ & $(0.005)$ \\
2006 & $0.009^{*}$ & $-0.009^{* *}$ & -0.006 \\
& $(0.005)$ & $(0.004)$ & $(0.004)$ \\
2008 & $0.009^{* *}$ & 0.001 & 0.001 \\
& $(0.004)$ & $(0.002)$ & $(0.002)$ \\
2010 & $0.007^{* * *}$ & -0.002 & -0.003 \\
& $(0.002)$ & $(0.002)$ & $(0.002)$ \\
2012 & $0.006^{* *}$ & -0.006 & -0.003 \\
& $(0.003)$ & $(0.004)$ & $(0.003)$ \\
2014 & 0.001 & 0.003 & 0.002 \\
& $(0.003)$ & $(0.003)$ & $(0.001)$ \\
2016 & $0.006^{* *}$ & & \\
& $(0.002)$ & & $0.003)$ \\
\hline
\end{tabular}

$\operatorname{Test} \operatorname{Cov}\left(\Delta \mathrm{y}_{\mathrm{t}+1}, \Delta \mathrm{c}_{\mathrm{t}}\right)=0$ para toda $\mathrm{t}$

p-value $25 \%$

$\operatorname{Test} \operatorname{Cov}\left(\Delta \mathrm{y}_{\mathrm{t}+2}, \Delta \mathrm{c}_{\mathrm{t}}\right)=0$ para toda $\mathrm{t}$

p-value $27 \%$

Nota: Errores estándar en paréntesis. Significancia: *** al 1\%; ** al 5\%; * al $10 \%$.

Estimación de la matriz de autocovarianzas basado en la ecuaciones 3 y 4 . Las variables $\Delta c_{t}$ y $\Delta y_{t}$ corresponden al incremento del consumo e ingreso no explicado por variables sociodemográficas, respectivamente.

Fuente: Estimación propia con datos del INEGI-ENIGH 2000-2016.

\footnotetext{
${ }^{20}$ Debido a que solo 3 años muestran resultados estadísticamente distintos de cero en la tabla 9.
} 
Para la región centro norte, y centro (tabla 11 y 12) la varianza del ingreso y del consumo se incrementa en el 2004 y hacia el 2014, para luego disminuir, lo mismo para la región sur (que alcanza su primer máximo en 2006, aunque no es significativo). Las covarianzas contemporáneas del incremento del ingreso y consumo no explicado, son estadísticamente significativas (primera columna). En la región centro norte, la covarianza del crecimiento del ingreso futuro con el crecimiento del consumo no explicado (segunda columna) no es significativa con excepción del año 2004, con resultados mixtos para la región centro y sur con cifras significativas para distintos años. Lo anterior apunta a que los choques temporales son cubiertos por las familias en las regiones norte y centro norte, con resultados no concluyentes para las otras regiones. Por su parte, para los choques permanentes existen indicios de posibles impactos al consumo para todas las regiones.

Tabla 11. Matrices de Covarianzas para el Crecimiento del Consumo y el Ingreso no explicado (ecuaciones 3 y 4), Región Centro Norte

Matriz de Autocovarianzas del Crecimiento del Consumo-Ingreso

\begin{tabular}{|c|c|c|c|}
\hline Año & $\operatorname{Cov}\left(\Delta y_{t}, \Delta c_{t}\right)$ & $\operatorname{Cov}\left(\Delta y_{t+1}, \Delta c_{t}\right)$ & $\operatorname{Cov}\left(\Delta \mathrm{y}_{\mathrm{t}}, \Delta \mathrm{c}_{\mathrm{t}+1}\right)$ \\
\hline \multirow[t]{2}{*}{2002} & $0.008^{*}$ & 0.000 & -0.002 \\
\hline & $(0.004)$ & $(0.004)$ & $(0.002)$ \\
\hline \multirow[t]{2}{*}{2004} & $0.015^{* * *}$ & $-0.004 *$ & -0.000 \\
\hline & (0.003) & $(0.002)$ & $(0.003)$ \\
\hline \multirow[t]{2}{*}{2006} & $0.005^{* *}$ & -0.002 & -0.001 \\
\hline & $(0.002)$ & $(0.001)$ & $(0.001)$ \\
\hline \multirow[t]{2}{*}{2008} & $0.004^{* *}$ & 0.001 & -0.001 \\
\hline & $(0.002)$ & $(0.002)$ & $(0.001)$ \\
\hline \multirow[t]{2}{*}{2010} & $0.004 * *$ & -0.002 & -0.001 \\
\hline & $(0.002)$ & (0.001) & $(0.002)$ \\
\hline \multirow[t]{2}{*}{2012} & $0.006 * *$ & -0.000 & $-0.007 * *$ \\
\hline & $(0.002)$ & $(0.002)$ & $(0.003)$ \\
\hline \multirow[t]{2}{*}{2014} & $0.009 * * *$ & -0.002 & -0.002 \\
\hline & $(0.003)$ & $(0.002)$ & $(0.002)$ \\
\hline \multirow[t]{2}{*}{2016} & $0.006 * * *$ & & \\
\hline & $(0.002)$ & & \\
\hline$f \Delta y_{t+1}$, & $=0$ para toda & & p-value $73 \%$ \\
\hline$\left(\Delta y_{t+2}\right.$ & $=0$ para toda & & p-value $60 \%$ \\
\hline
\end{tabular}

Nota: Errores estándar en paréntesis. Significancia: *** al 1\%; ** al 5\%; * al 10\%.

Estimación de la matriz de autocovarianzas basado en la ecuaciones 3 y 4 . Las variables $\Delta c_{t}$ y $\Delta y_{t}$ corresponden al incremento del consumo e ingreso no explicado por variables sociodemográficas, respectivamente.

Fuente: Estimación propia con datos del INEGI-ENIGH 2000-2016. 
Tabla 12. Matrices de Covarianzas para el Crecimiento del Consumo y el Ingreso no explicado (ecuaciones 3 y 4), Región Centro

Matriz de Autocovarianzas del Crecimiento del Consumo-Ingreso

\begin{tabular}{rlll}
\hline Año & $\operatorname{Cov}\left(\Delta \mathrm{y}_{\mathrm{t}} \Delta \mathrm{c}_{\mathrm{t}}\right)$ & $\operatorname{Cov}\left(\Delta \mathrm{y}_{\mathrm{t}+1}, \Delta \mathrm{c}_{\mathrm{t}}\right)$ & $\operatorname{Cov}\left(\Delta \mathrm{y}_{\mathrm{t}}, \Delta \mathrm{c}_{\mathrm{t}+1}\right)$ \\
\hline 2002 & $0.017^{* * *}$ & $-0.006^{*}$ & -0.007 \\
& $(0.004)$ & $(0.003)$ & $(0.005)$ \\
2004 & $0.043^{* *}$ & -0.003 & -0.001 \\
& $(0.017)$ & $(0.003)$ & $(0.004)$ \\
2006 & $0.005^{* * *}$ & $-0.003^{* * *}$ & -0.001 \\
& $(0.001)$ & $(0.001)$ & $(0.002)$ \\
2008 & $0.007^{* * *}$ & 0.001 & $-0.002^{*}$ \\
& $(0.002)$ & $(0.002)$ & $(0.001)$ \\
2010 & $0.004^{* * *}$ & -0.001 & $-0.003^{* *}$ \\
& $(0.001)$ & $(0.001)$ & $(0.001)$ \\
2012 & 0.005 & $-0.007^{* *}$ & -0.005 \\
& $(0.003)$ & $(0.004)$ & $(0.004)$ \\
2014 & $0.009^{* * *}$ & $-0.002^{*}$ & -0.002 \\
& $(0.003)$ & $(0.001)$ & $(0.002)$ \\
2016 & $0.006^{* * *}$ & & \\
& $(0.002)$ & & $\mathrm{P}$ \\
\hline
\end{tabular}

$\begin{array}{ll}\text { Test } \operatorname{Cov}\left(\Delta y_{t+1}, \Delta c_{t}\right)=0 \text { para toda } t & p \text {-value } 23 \% \\ \text { Test } \operatorname{Cov}\left(\Delta y_{t+2}, \Delta c_{t}\right)=0 \text { para toda } t & p \text {-value } 57 \%\end{array}$

Nota: Errores estándar en paréntesis. Significancia: *** al 1\%; ** al 5\%; * al $10 \%$.

Estimación de la matriz de autocovarianzas basado en la ecuaciones 3 y 4 . Las variables $\Delta c_{t}$ y $\Delta y_{t}$ corresponden al incremento del consumo e ingreso no explicado por variables sociodemográficas, respectivamente.

Fuente: Estimación propia con datos del INEGI-ENIGH 2000-2016. 


\section{Tabla 13. Matrices de Covarianzas para el Crecimiento del Consumo y el Ingreso no explicado (ecuaciones 3 y 4), Región Sur}

Matriz de Autocovarianzas del Crecimiento del Consumo-Ingreso

\begin{tabular}{rlll}
\hline Año & $\operatorname{Cov}\left(\Delta \mathrm{y}_{\mathrm{t}}, \Delta \mathrm{c}_{\mathrm{t}}\right)$ & $\operatorname{Cov}\left(\Delta \mathrm{y}_{\mathrm{t}+1}, \Delta \mathrm{c}_{\mathrm{t}}\right)$ & $\operatorname{Cov}\left(\Delta \mathrm{y}_{\mathrm{t}}, \Delta \mathrm{c}_{\mathrm{t}+1}\right)$ \\
\hline 2002 & $0.008^{* * *}$ & $0.005^{*}$ & 0.002 \\
& $(0.002)$ & $(0.003)$ & $(0.002)$ \\
2004 & $0.013^{* * *}$ & $-0.003^{*}$ & -0.001 \\
& $(0.004)$ & $(0.002)$ & $(0.003)$ \\
2006 & 0.017 & $-0.008^{* *}$ & -0.004 \\
& $(0.011)$ & $(0.003)$ & $(0.004)$ \\
2008 & $0.009 * * *$ & $-0.007^{* *}$ & $-0.004^{* *}$ \\
& $(0.003)$ & $(0.003)$ & $(0.002)$ \\
2010 & $0.008^{* * *}$ & 0.001 & $-0.003^{*}$ \\
& $(0.002)$ & $(0.002)$ & $(0.002)$ \\
2012 & $0.006 * *$ & $-0.008^{* *}$ & $-0.011^{* * *}$ \\
& $(0.003)$ & $(0.003)$ & $(0.003)$ \\
2014 & $0.012^{* * *}$ & -0.001 & -0.001 \\
& $(0.003)$ & $(0.002)$ & $(0.002)$ \\
2016 & $0.006 * *$ & & \\
& $(0.002)$ & & $0-v a 1487 \%$ \\
\hline
\end{tabular}

Test $\operatorname{Cov}\left(\Delta \mathrm{y}_{\mathrm{t}+1}, \Delta \mathrm{c}_{\mathrm{t}}\right)=0$ para toda $\mathrm{t}$

p-value $7 \%$

Test $\operatorname{Cov}\left(\Delta \mathrm{y}_{\mathrm{t}+2}, \Delta \mathrm{c}_{\mathrm{t}}\right)=0$ para toda $\mathrm{t}$

p-value $38 \%$

Nota: Errores estándar en paréntesis. Significancia: *** al 1\%; ** al 5\%; * al 10\%.

Estimación de la matriz de autocovarianzas basado en la ecuaciones 3 y 4 . Las variables $\Delta c_{t}$ y $\Delta y_{t}$ corresponden al incremento del consumo e ingreso no explicado por variables sociodemográficas, respectivamente.

Fuente: Estimación propia con datos del INEGI-ENIGH 2000-2016.

Resultados formales de los coeficientes de aseguramiento de los choques del ingreso al consumo.

A diferencia de Blundell et al. (2008) y por limitaciones de la base de datos, asumiremos que los coeficientes de aseguramiento del consumo ante choques en el ingreso permanente $(\phi)$ y para choques en el ingreso transitorio $(\psi)$ son constantes a lo largo del periodo de estudio. ${ }^{21}$ Como se mencionó, esta metodología nos permite probar si la hipótesis del ingreso permanente $(\phi=1, y \psi=0)$ se cumple, o la de mercados completos $(\phi=0, y \psi=0)$, o la de ahorro precautorio, con un coeficiente $0<\phi<1$.

En la tabla 14 se muestran los resultados para ambos coeficientes a nivel nacional y para cada una de las regiones, el coeficiente del impacto del consumo debido a choques permanentes en el ingreso $(\phi)$ resultó significativo con un coeficiente de 0.68 , es decir, ante una reducción

\footnotetext{
${ }^{21}$ Dado que se analiza un periodo relativamente corto de tiempo dicho supuesto no es tan restrictivo.
} 
(aumento) del 10\% en el ingreso permanente, el consumo se reduciría (incrementaría) en un $6.8 \%$. Por su parte, el resultado para los choques transitorios no es significativo a nivel nacional ni para ninguna de las regiones, lo anterior va en línea con los resultados del análisis de las covarianzas entre los crecimientos de los choques no explicados del ingreso y el consumo realizado en las tablas 8 y 9. Cabe señalar que dicho coeficiente sugiere un aseguramiento parcial del consumo ante choques permanentes en el ingreso y es similar al estimado para Estados Unidos por Blundell et al. (2008), quienes obtienen un coeficiente de 0.64. Dicho resultado pudiera parecer sorprendente dado que la economía mexicana cuenta con un menor nivel de desarrollo económico. Sin embargo, esto no implica que los hogares utilicen los mismos medios para hacer frente a un choque permanente en su ingreso, o que los hogares en los dos países cuenten con los mismos niveles de ingreso y de consumo. En específico, los hogares en economías más avanzadas tienden a emplear mecanismos formales (con acceso al sistema financiero), mientras que en países en vías de desarrollo algunos hogares no tienen acceso al sistema financiero y pueden recurrir a medios informales (familiares, amistades, etc.)

Así los resultados a nivel nacional sugieren que las cohortes mexicanas estudiadas en este documento aseguran parcialmente los choques no esperados en el ingreso permanente, ya que el coeficiente es menor a uno pero estadísticamente diferente de cero. Asimismo, las estimaciones indican que el consumo no registra cambios ante choques temporales no esperados en los ingresos. Dichos resultados son consistentes con la hipótesis de ahorro precautorio, en otras palabras, al enfrentar mercados incompletos, las familias no pueden diversificar los impactos, por lo que se ven forzadas a un aseguramiento ad-hoc (ahorro para imprevistos, red familiar o de amistades, o acceso a créditos). No obstante, las estimaciones no descartan la hipótesis del ingreso permanente a nivel nacional, debido a que $\phi=1$ cae dentro del intervalo de confianza del 95 por ciento, así como $\psi=0$. 
Tabla 14. Estimación de los coeficientes de los choques permanente y transitorio (ecuación 4) por mínima distancia

\begin{tabular}{cccccc}
\hline Parámetro & Nacional & Norte & $\begin{array}{c}\text { Centro } \\
\text { Norte }\end{array}$ & Centro & Sur \\
\hline$\phi$ & $0.679 * *$ & 0.675 & $0.792^{* *}$ & 0.750 & $0.917 * *$ \\
$\begin{array}{c}\text { Choque } \\
\text { permanente }\end{array}$ & $(0.298)$ & $(1.032)$ & $(0.337)$ & $(0.912)$ & $(0.448)$ \\
$\psi$ & 0.0874 & -0.115 & -0.201 & 0.217 & -0.220 \\
$\begin{array}{c}\text { Choque } \\
\text { transitorio }\end{array}$ & $(0.233)$ & $(0.300)$ & $(0.222)$ & $(0.202)$ & $(0.152)$ \\
\hline
\end{tabular}

Nota: Errores estándar en paréntesis. Significancia: *** al 1\%; ** al 5\%; * al 10\%. Estimación utilizando la metodología basada en Blundell et al. (2008) Apéndice C.

Fuente: Estimación propia con datos del INEGI-ENIGH 2000-2016.

Dichos impactos permanentes pueden tener efectos, tanto positivos como negativos en la movilidad en el ingreso. Krebs et al. (2013) estima que gran parte de la movilidad convergente (convergencia en los ingresos de individuos con las mismas características) se debe a choques transitorios en el ingreso, que en nuestro caso son absorbidos de manera intertemporal por el consumo.

A nivel regional, se estiman coeficientes con niveles similares a los del nivel nacional para los choques permanentes en el ingreso en las regiones norte, centro norte y centro, y de mayor magnitud en el sur, si bien con significancia estadística solo en las regiones centro norte y sur. $^{22}$ En referencia a los choques temporales, el coeficiente no resulta estadísticamente significativo en ninguna de las regiones, lo que sugiere que los hogares son capaces de suavizar su consumo ante cambios temporales en su ingreso.

\section{Conclusiones}

En el presente documento de investigación, se analizaron los efectos de choques inesperados en el ingreso de los hogares sobre el consumo de bienes no durables, tanto temporales como permanentes, a nivel nacional y para cada una de las regiones de México. Los resultados de la investigación arrojan que a nivel nacional existe un impacto parcial en el consumo de las familias ante choques permanentes en el ingreso estadísticamente significativo $(\phi=67.9 \%)$.

\footnotetext{
${ }^{22}$ Los resultados a nivel regional deben tomarse con precaución, debido al número reducido de muestra de hogares por cohorte en algunas regiones.
} 
Es decir, por cada 100 pesos de impacto en el ingreso permanente, las familias mexicanas ajustan su consumo en 67.9 pesos durante el periodo de análisis. Por su parte, el coeficiente de los choques transitorios en el ingreso $(\psi)$ no es estadísticamente significativo, lo que podría sugerir que las familias en México son capaces de suavizar dichos impactos.

Así, los resultados sugieren para el caso de México: i) la magnitud del impacto en el consumo ante choques permanentes en el ingreso es acorde a la hipótesis de aseguramiento parcial, debido a que su estimador puntual $(\phi)$ es menor a la unidad y el coeficiente correspondiente del impacto en el consumo ante choques transitorios en el ingreso $(\psi)$ no es estadísticamente significativo; ii) la evidencia también es consistente con la hipótesis de ingreso permanente, debido a que el coeficiente del impacto en el consumo ante choques en el ingreso permanente $\phi=1$ está contenido dentro del intervalo de confianza, al igual que el estimador de efectos en el consumo ante choques transitorios $(\psi=0)$; iii) se descarta la hipótesis de mercados completos (aseguramiento total), debido a que el estimador de los impactos en el consumo ante choques permanentes en el ingreso es estadísticamente distinto de cero.

A nivel regional, los coeficientes del efecto del choque permanente en el ingreso sobre el consumo son similares al estimado a nivel nacional en las regiones norte, centro norte y centro, y de mayor magnitud en el sur, si bien con significancia estadística solo en las regiones centro norte y sur. En referencia a los choques temporales, el coeficiente del efecto del choque al ingreso sobre el consumo no resulta estadísticamente significativo a nivel regional, lo que sugiere un suavizamiento del consumo por parte de los hogares ante cambios temporales en su ingreso.

En suma, estos resultados sugieren que, a nivel nacional, los hogares mexicanos ven afectado su consumo ante choques inesperados en sus ingresos permanentes, pero que suavizan su consumo ante cambios no esperados y temporales en sus ingresos. Cabe señalar que se analiza un periodo de la economía mexicana en el que, si bien se registraron variaciones en el ingreso, dichos cambios no fueron tan grandes comparados con el choque observado en los ingresos en 1995 o más recientemente en 2020 como consecuencia de la pandemia de COVID-19. Aunado a lo anterior, el análisis propuesto se fortalecería con la presencia de 
datos longitudinales para capturar cambios en los patrones de consumo, los cuales no disponemos a la fecha de la elaboración del presente documento. 


\section{Bibliografía}

Agarwal, S. \& Qian, W. (2014); Consumption and Debt Response to Unanticipated Income Shocks: Evidence from a Natural Experiment in Singapore. American Economic Review, 104(12).

Attanasio, O. \& Székely, M. (1998); Household Savings and Income Distribution in Mexico, Department Publications 4152, Inter-American Development Bank, Research Department.

Attanasio, O. \& Székely, M. (2004); Wage shocks and consumption variability in Mexico during the 1990s. Journal of Development Economics, 73, 1-25.

Browning M. \& Crossley, T. (2001); Unemployment Insurance Benefit Levels and Consumption Changes. Journal of Public Economics, 80.

Blundell, R. Pistaferri, L. \& Preston, I. (2008); Consumption Inequality and Partial Insurance, American Economic Review, 98(5), 1887-1921.

Diario Oficial de la Federación (2010); Lineamientos y criterios generales para la definición, identificación y medición de la pobreza; 16 de Junio de 2010.

Gruber, J. (1997); The Consumption Smoothing Benefits of Unemployment Insurance. The American Economic Review, 87(1).

Hayashi, F. (1985); The Permanent Income Hypothesis and Consumption Durability: Analysis Based on Japanese Panel Data. Quarterly Journal of Economics, 100.

Heath, J. (2011); Identificación de los Ciclos Económicos en México: 30 años de evidencia; Realidad, Datos y Espacio Revista Internacional de Estadística y Geografía, INEGI, 2(2)

Jappelli, T. \& Pistaferri, L. (2010); The Consumption Response to Income Changes, The Annual Review of Economics, 2, 479-506.

Jappelli, T. \& Pistaferri, L. (2017); The Economics of Consumption, Theory and Evidence. Oxford University Press. 
Krebs, T; Krishna, P. \& Maloney, W. (2019); Income Mobility, Income Risk, and Welfare. The World Bank Economic Review, 33(2).

Kreinin, M. (1961); Windfall Income and Consumption: Additional Evidence. The American Economic Review, 51(3).

Paxon, C. (1993); Consumption and Income Seasonality in Thailand, Journal of Political Economy, 101, 39-72.

Primiceri, G. y van Rens, T. (2009); Heterogeneous life-cycle profiles, income risk and consumption inequality, Journal of Monetary Economics, 56, 20-39.

Teruel, G., Rubalcava, L. \& Santana, A. (2005); Escalas de Equivalencia para México; Documento de Investigación 23, Secretaría de Desarrollo Social.

Wolpin, K. (1982); A New Test of the Permanent Income Hypothesis: The Impact of Weather on the Income and Consumption of Farm Households in India, International Economic Review, 23(3). 
8. Anexos

Tabla A.1. Matrices de Covarianzas para el Crecimiento del Ingreso-Consumo, Región Norte

Matriz de Autocovarianzas del Crecimiento del Ingreso

\begin{tabular}{rlll}
\multicolumn{5}{c}{ Matriz de Autocovarianzas del Crecimiento del Ingreso } \\
\hline Año & $\operatorname{Var}\left(\Delta \mathrm{y}_{\mathrm{t}}\right)$ & $\operatorname{Cov}\left(\Delta \mathrm{y}_{\mathrm{t}+1}, \Delta \mathrm{y}_{\mathrm{t}}\right)$ & $\operatorname{Cov}\left(\Delta \mathrm{y}_{\mathrm{t}+2}, \Delta \mathrm{y}_{\mathrm{t}}\right)$ \\
\hline 2002 & $0.023^{* *}$ & -0.003 & 0.002 \\
& $(0.009)$ & $(0.005)$ & $(0.003)$ \\
2004 & $0.022^{* * *}$ & 0.002 & -0.004 \\
& $(0.005)$ & $(0.004)$ & $(0.003)$ \\
2006 & $0.010^{* * *}$ & $-0.005^{* *}$ & $-0.003^{*}$ \\
& $(0.003)$ & $(0.002)$ & $(0.002)$ \\
2008 & $0.008^{* * *}$ & 0.000 & -0.000 \\
& $(0.002)$ & $(0.001)$ & $(0.001)$ \\
2010 & $0.008^{* * *}$ & $-0.004^{* *}$ & -0.001 \\
& $(0.002)$ & $(0.002)$ & $(0.002)$ \\
2012 & $0.009^{* * *}$ & $-0.009^{* * *}$ & 0.003 \\
& $(0.003)$ & $(0.003)$ & $(0.002)$ \\
2014 & $0.012^{* * *}$ & $-0.004^{*}$ & \\
& $(0.002)$ & $(0.002)$ & \\
2016 & $0.014^{* * *}$ & & \\
& $(0.003)$ & & \\
\hline
\end{tabular}

Matriz de Autocovarianzas del Crecimiento del Consumo

\begin{tabular}{rlll}
\hline Año & $\operatorname{Var}\left(\Delta \mathrm{c}_{\mathrm{t}}\right)$ & $\operatorname{Cov}\left(\Delta \mathrm{c}_{\mathrm{t}+1}, \Delta \mathrm{c}_{\mathrm{t}}\right)$ & $\operatorname{Cov}\left(\Delta \mathrm{c}_{\mathrm{t}+2}, \Delta \mathrm{c}_{\mathrm{t}}\right)$ \\
\hline 2002 & $0.014^{* * *}$ & 0.002 & -0.001 \\
& $(0.004)$ & $(0.004)$ & $(0.003)$ \\
2004 & $0.023^{* * *}$ & 0.004 & $-0.016^{* * *}$ \\
& $(0.005)$ & $(0.005)$ & $(0.005)$ \\
2006 & $0.023^{* * *}$ & $-0.019 * *$ & -0.001 \\
& $(0.008)$ & $(0.008)$ & $(0.004)$ \\
2008 & $0.027^{* * *}$ & -0.003 & 0.001 \\
& $(0.007)$ & $(0.004)$ & $(0.002)$ \\
2010 & $0.014 * * *$ & $-0.006 * *$ & -0.001 \\
& $(0.003)$ & $(0.002)$ & $(0.002)$ \\
2012 & $0.014 * * *$ & -0.008 & -0.000 \\
& $(0.004)$ & $(0.006)$ & $(0.002)$ \\
2014 & $0.015^{* * *}$ & $-0.005^{* * *}$ & \\
& $(0.004)$ & $(0.001)$ & \\
2016 & $0.009 * * *$ & & \\
& $(0.002)$ & & \\
& \multirow{2}{*}{2016} & & \\
\hline
\end{tabular}

Nota: Errores estándar en paréntesis. Significancia: *** al 1\%; ** al 5\%; * al 10\%.

Fuente: Estimación propia con datos del INEGI-ENIGH 2008-2016. 
Tabla A.2. Matrices de Covarianzas para el Crecimiento del Ingreso-Consumo, Región Centro Norte

Matriz de Autocovarianzas del Crecimiento del Ingreso

\begin{tabular}{rccc}
\hline Año & $\operatorname{Var}\left(\Delta \mathrm{y}_{\mathrm{t}}\right)$ & $\operatorname{Cov}\left(\Delta \mathrm{y}_{\mathrm{t}+1}, \Delta \mathrm{y}_{\mathrm{t}}\right)$ & $\operatorname{Cov}\left(\Delta \mathrm{y}_{\mathrm{t}+2}, \Delta \mathrm{y}_{\mathrm{t}}\right)$ \\
\hline 2002 & $0.014^{* *}$ & -0.006 & 0.000 \\
& $(0.006)$ & $(0.005)$ & $(0.002)$ \\
2004 & $0.022^{* * *}$ & $-0.008^{* * *}$ & 0.002 \\
& $(0.005)$ & $(0.002)$ & $(0.002)$ \\
2006 & $0.009^{* * *}$ & $-0.005^{* * *}$ & 0.000 \\
& $(0.002)$ & $(0.001)$ & $(0.002)$ \\
2008 & $0.010^{* * *}$ & -0.001 & -0.002 \\
& $(0.003)$ & $(0.001)$ & $(0.002)$ \\
2010 & $0.008^{* * *}$ & $-0.005^{* * *}$ & -0.002 \\
& $(0.002)$ & $(0.001)$ & $(0.002)$ \\
2012 & $0.012^{* * *}$ & $-0.009^{* *}$ & 0.003 \\
& $(0.003)$ & $(0.003)$ & $(0.002)$ \\
2014 & $0.019^{* * *}$ & $-0.005^{* *}$ & \\
& $(0.005)$ & $(0.002)$ & \\
2016 & $0.010^{* * *}$ & & \\
& $(0.002)$ & & \\
& & & \\
& & &
\end{tabular}

\begin{tabular}{rccc}
\multicolumn{5}{c}{ Matriz de Autocovarianzas del Crecimiento del Consumo } \\
\hline Año & $\operatorname{Var}\left(\Delta \mathrm{c}_{\mathrm{t}}\right)$ & $\operatorname{Cov}\left(\Delta \mathrm{c}_{\mathrm{t}+1}, \Delta \mathrm{c}_{\mathrm{t}}\right)$ & $\operatorname{Cov}\left(\Delta \mathrm{c}_{\mathrm{t}+2}, \Delta \mathrm{c}_{\mathrm{t}}\right)$ \\
\hline 2002 & $0.015^{* * *}$ & -0.000 & -0.004 \\
& $(0.003)$ & $(0.002)$ & $(0.003)$ \\
2004 & $0.013^{* * *}$ & -0.002 & -0.001 \\
& $(0.003)$ & $(0.002)$ & $(0.002)$ \\
2006 & $0.011^{* * *}$ & $-0.007^{* * *}$ & -0.001 \\
& $(0.003)$ & $(0.002)$ & $(0.002)$ \\
2008 & $0.012^{* * *}$ & -0.001 & -0.001 \\
& $(0.002)$ & $(0.001)$ & $(0.002)$ \\
2010 & $0.009^{* * *}$ & -0.004 & -0.002 \\
& $(0.002)$ & $(0.002)$ & $(0.002)$ \\
2012 & $0.011^{* * *}$ & $-0.006^{* * *}$ & 0.002 \\
& $(0.003)$ & $(0.002)$ & $(0.001)$ \\
2014 & $0.016^{* * *}$ & $-0.007^{*}$ & \\
& $(0.003)$ & $(0.003)$ & \\
2016 & $0.011^{* * *}$ & & \\
& $(0.003)$ & & \\
\hline
\end{tabular}

Nota: Errores estándar en paréntesis. Significancia: *** al 1\%; ** al 5\%; * al 10\%.

Fuente: Estimación propia con datos del INEGI-ENIGH 2008-2016. 
Tabla A.3. Matrices de Covarianzas para el Crecimiento del Ingreso-Consumo, Región Centro

Matriz de Autocovarianzas del Crecimiento del Ingreso

\begin{tabular}{rlll}
\hline Año & \multicolumn{1}{c}{$\operatorname{Var}\left(\Delta \mathrm{y}_{\mathrm{t}}\right)$} & $\operatorname{Cov}\left(\Delta \mathrm{y}_{\mathrm{t}+1}, \Delta \mathrm{y}_{\mathrm{t}}\right)$ & $\operatorname{Cov}\left(\Delta \mathrm{y}_{\mathrm{t}+2}, \Delta \mathrm{y}_{\mathrm{t}}\right)$ \\
\hline 2002 & $0.035^{* * *}$ & -0.010 & -0.000 \\
& $(0.007)$ & $(0.006)$ & $(0.004)$ \\
2004 & $0.056^{* * *}$ & $-0.007^{* *}$ & -0.001 \\
& $(0.020)$ & $(0.003)$ & $(0.002)$ \\
2006 & $0.009^{* * *}$ & $-0.005^{* *}$ & 0.001 \\
& $(0.003)$ & $(0.002)$ & $(0.001)$ \\
2008 & $0.009^{* * *}$ & $-0.003^{* *}$ & $-0.003^{*}$ \\
& $(0.002)$ & $(0.001)$ & $(0.001)$ \\
2010 & $0.007^{* * *}$ & $-0.003^{*}$ & 0.000 \\
& $(0.002)$ & $(0.001)$ & $(0.001)$ \\
2012 & $0.008^{* * *}$ & $-0.007^{* *}$ & 0.003 \\
& $(0.002)$ & $(0.003)$ & $(0.002)$ \\
2014 & $0.012^{* * *}$ & $-0.004^{* *}$ & \\
& $(0.003)$ & $(0.002)$ & \\
2016 & $0.009^{* * *}$ & & \\
& $(0.002)$ & & \\
& & &
\end{tabular}

Matriz de Autocovarianzas del Crecimiento del Consumo

\begin{tabular}{rlll}
\hline Año & $\operatorname{Var}\left(\Delta \mathrm{c}_{\mathrm{t}}\right)$ & $\operatorname{Cov}\left(\Delta \mathrm{c}_{\mathrm{t}+1}, \Delta \mathrm{c}_{\mathrm{t}}\right)$ & $\operatorname{Cov}\left(\Delta \mathrm{c}_{\mathrm{t}+2}, \Delta \mathrm{c}_{\mathrm{t}}\right)$ \\
\hline 2002 & $0.015^{* * *}$ & $-0.007^{* *}$ & 0.002 \\
& $(0.004)$ & $(0.003)$ & $(0.002)$ \\
2004 & $0.042^{* * *}$ & -0.001 & $-0.009^{* *}$ \\
& $(0.014)$ & $(0.003)$ & $(0.004)$ \\
2006 & $0.006^{* * *}$ & $-0.003^{*}$ & 0.000 \\
& $(0.001)$ & $(0.001)$ & $(0.001)$ \\
2008 & $0.016^{* * *}$ & -0.001 & -0.001 \\
& $(0.004)$ & $(0.001)$ & $(0.002)$ \\
2010 & $0.005^{* * *}$ & $-0.003^{* *}$ & $-0.002^{*}$ \\
& $(0.001)$ & $(0.001)$ & $(0.001)$ \\
2012 & $0.012^{* *}$ & -0.010 & -0.002 \\
& $(0.005)$ & $(0.006)$ & $(0.002)$ \\
2014 & $0.016^{* * *}$ & $-0.004^{*}$ & \\
& $(0.005)$ & $(0.002)$ & \\
& $0.012^{* * *}$ & & \\
& $(0.002)$ & & \\
\hline
\end{tabular}

Nota: Errores estándar en paréntesis. Significancia: *** al 1\%; ** al 5\%; * al 10\%.

Fuente: Estimación propia con datos del INEGI-ENIGH 2008-2016. 
Tabla A.4. Matrices de Covarianzas para el Crecimiento del Ingreso-Consumo, Región Sur

Matriz de Autocovarianzas del Crecimiento del Ingreso

\begin{tabular}{clll}
\multicolumn{5}{c}{ Matriz de Autocovarianzas del Crecimiento del Ingreso } \\
\hline Año & \multicolumn{1}{c}{$\operatorname{Var}\left(\Delta \mathrm{y}_{\mathrm{t}}\right)$} & $\operatorname{Cov}\left(\Delta \mathrm{y}_{\mathrm{t}+1}, \Delta \mathrm{y}_{\mathrm{t}}\right)$ & $\operatorname{Cov}\left(\Delta \mathrm{y}_{\mathrm{t}+2}, \Delta \mathrm{y}_{\mathrm{t}}\right)$ \\
\hline 2002 & $0.012^{* * *}$ & 0.003 & -0.002 \\
& $(0.004)$ & $(0.003)$ & $(0.003)$ \\
2004 & $0.028^{* * *}$ & $-0.007^{* * *}$ & 0.005 \\
& $(0.006)$ & $(0.002)$ & $(0.003)$ \\
2006 & $0.021^{* *}$ & $-0.009^{* *}$ & $0.005^{*}$ \\
& $(0.010)$ & $(0.003)$ & $(0.002)$ \\
2008 & $0.017^{* * *}$ & $-0.010^{* * *}$ & -0.002 \\
& $(0.004)$ & $(0.003)$ & $(0.002)$ \\
2010 & $0.014^{* * *}$ & -0.001 & -0.002 \\
& $(0.003)$ & $(0.001)$ & $(0.001)$ \\
2012 & $0.011^{* * *}$ & $-0.013^{* * *}$ & $0.002^{*}$ \\
& $(0.003)$ & $(0.002)$ & $(0.001)$ \\
2014 & $0.017^{* * *}$ & $-0.005^{* *}$ & \\
& $(0.003)$ & $(0.002)$ & \\
2016 & $0.011^{* * *}$ & & \\
& $(0.003)$ & &
\end{tabular}

Matriz de Autocovarianzas del Crecimiento del Consumo

\begin{tabular}{|c|c|c|c|}
\hline Año & $\operatorname{Var}\left(\Delta c_{t}\right)$ & $\operatorname{Cov}\left(\Delta c_{t+1}, \Delta c_{t}\right)$ & $\operatorname{Cov}\left(\Delta c_{t+2}, \Delta c_{t}\right)$ \\
\hline \multirow[t]{2}{*}{2002} & $0.009 * * *$ & 0.001 & -0.001 \\
\hline & $(0.002)$ & $(0.002)$ & $(0.002)$ \\
\hline \multirow[t]{2}{*}{2004} & $0.016 * * *$ & -0.002 & $-0.008 * *$ \\
\hline & $(0.003)$ & $(0.002)$ & $(0.003)$ \\
\hline \multirow[t]{2}{*}{2006} & $0.023 *$ & $-0.012^{* *}$ & 0.003 \\
\hline & $(0.012)$ & $(0.004)$ & $(0.003)$ \\
\hline \multirow[t]{2}{*}{2008} & $0.022 * * *$ & $-0.009 * *$ & -0.001 \\
\hline & $(0.005)$ & $(0.003)$ & $(0.002)$ \\
\hline \multirow[t]{2}{*}{2010} & $0.013^{* * *}$ & $-0.004^{*}$ & $-0.006 * * *$ \\
\hline & $(0.003)$ & $(0.002)$ & $(0.002)$ \\
\hline \multirow[t]{2}{*}{2012} & $0.011^{* *}$ & $-0.011^{*}$ & -0.002 \\
\hline & $(0.004)$ & $(0.005)$ & $(0.001)$ \\
\hline \multirow[t]{2}{*}{2014} & $0.017^{* * *}$ & $-0.003^{*}$ & \\
\hline & $(0.005)$ & $(0.002)$ & \\
\hline \multirow[t]{2}{*}{2016} & $0.014 * * *$ & & \\
\hline & $(0.003)$ & & \\
\hline
\end{tabular}

Nota: Errores estándar en paréntesis. Significancia: *** al 1\%; ** al 5\%; * al $10 \%$.

Fuente: Estimación propia con datos del INEGI-ENIGH 2008-2016. 
Tabla A.5. Resultados de las Regresiones del Ingreso y el Consumo de No Durables con las Variables Demográficas $\mathbf{Z}$

\begin{tabular}{ccc}
\hline & $\begin{array}{c}(1) \\
\text { ln(Ingreso } \\
\text { hogar) }\end{array}$ & $\begin{array}{c}(2) \\
\text { n(Consumo } \\
\text { no durable) }\end{array}$ \\
\hline Jefe de Familia tiene acceso a & $0.461^{* * *}$ & $0.471^{* * *}$ \\
seguridad social & $(0.068)$ & $(0.062)$ \\
Hogar vive en una localidad & $0.279^{* * *}$ & $0.257^{* * *}$ \\
urbana & $(0.080)$ & $(0.073)$ \\
Número de menores de 10 años & -0.042 & -0.020 \\
promedio. & $(0.029)$ & $(0.027)$ \\
Número de adultos & $0.176^{* * *}$ & $0.135^{* * *}$ \\
promedio. & $(0.035)$ & $(0.032)$ \\
Número de perceptores & $0.051^{* * *}$ & $0.152^{* * *}$ \\
promedio. & $(0.013)$ & $(0.011)$ \\
Horas trabajadas por hogar & -0.000 & $-0.002^{* *}$ \\
promedio. & $(0.001)$ & $(0.001)$ \\
Años de educación del & $0.016^{* * *}$ & $0.019 * * *$ \\
cónyugue & $(0.005)$ & $(0.005)$ \\
Efectos Fijos Cohorte & $\mathrm{Si}$ & $\mathrm{Si}$ \\
Efectos Fijos Año & $\mathrm{Si}$ & $\mathrm{Si}$ \\
Observaciones & 496 & 496 \\
$\mathrm{R}^{2}$ & 0.972 & 0.958 \\
\hline
\end{tabular}

Nota: Errores estándar en paréntesis. Significancia: *** al 1\%; ** al 5\%; * al 10\%.

Fuente: Estimación propia con datos del INEGI-ENIGH 2008-2016. 
Tabla A.6. Comparativo de Promedios por Factor de Expansión y Panel Sintético de Variables de Ingreso, Gasto No Durable, Miembros Promedio del Hogar y Número de Perceptores Promedio por Nivel Educativo y Levantamiento de ENIGH 2000-2016

\begin{tabular}{|c|c|c|c|c|c|c|c|c|c|}
\hline \multirow[b]{2}{*}{$\begin{array}{c}\text { Levantamiento } \\
\text { ENIGH }\end{array}$} & \multirow[b]{2}{*}{ Educación Jefe de Familia } & \multicolumn{4}{|c|}{ Promedios Utilizando Factor de Expansión } & \multicolumn{4}{|c|}{ Promedios Celdas Panel Sintético } \\
\hline & & \begin{tabular}{|c|} 
Promedio \\
Ingreso
\end{tabular} & \begin{tabular}{|c|} 
Promedio \\
Gasto No \\
Durable
\end{tabular} & $\begin{array}{c}\text { Numero de } \\
\text { miembros } \\
\text { promedio }\end{array}$ & $\begin{array}{c}\text { Numero de } \\
\text { perceptores } \\
\text { promedio }\end{array}$ & \begin{tabular}{|c|}
$\begin{array}{c}\text { Promedio } \\
\text { Ingreso }\end{array}$ \\
\end{tabular} & \begin{tabular}{|c|} 
Promedio \\
Gasto No \\
Durable
\end{tabular} & $\begin{array}{c}\text { Numero de } \\
\text { mi embros } \\
\text { promedio }\end{array}$ & \begin{tabular}{|c|} 
Numero de \\
perceptores \\
promedio
\end{tabular} \\
\hline \multirow{7}{*}{2000} & Sin Instrucción & 18,995 & 9,392 & 5.0 & 1.6 & 21,639 & 10,580 & 4.9 & 1.6 \\
\hline & de 1 a 4 años (Primaria) & 20,041 & 10,248 & 4.9 & 1.9 & 21,413 & 10,853 & 4.8 & 1.8 \\
\hline & 5to y 6 to de primaria & 26,567 & 12,143 & 4.5 & 1.8 & 29,700 & 13,428 & 4.4 & 1.7 \\
\hline & Secundaria & 39,610 & 16,321 & 3.9 & 1.6 & 38,582 & 15,211 & 3.9 & 1.6 \\
\hline & 1ro y 2 do de Preparatoria & 47,633 & 18,404 & 4.2 & 1.7 & 41,834 & 16,632 & 4.1 & 1.6 \\
\hline & Preparatoria & 79,675 & 27,005 & 3.7 & 1.5 & 89,151 & 28,846 & 3.7 & 1.5 \\
\hline & Superior & 67,580 & 23,734 & 3.9 & 1.6 & 74,549 & 25,233 & 3.8 & 1.6 \\
\hline \multirow{7}{*}{2002} & Sin Instrucción & 17,669 & 8,646 & 4.7 & 1.7 & 18,127 & 8,937 & 5.0 & 1.8 \\
\hline & de 1 a 4 años (Primaria) & 21,059 & 9,863 & 4.8 & 1.8 & 22,423 & 10,255 & 5.0 & 1.9 \\
\hline & 5to y 6 to de primaria & 26,630 & 12,370 & 4.5 & 1.8 & 28,448 & 12,701 & 4.5 & 1.8 \\
\hline & Secundaria & 32,904 & 13,803 & 3.7 & 1.6 & 32,978 & 13,483 & 3.9 & 1.7 \\
\hline & 1ro y 2 do de Preparatoria & 38,589 & 14,553 & 3.9 & 1.7 & 39,835 & 15,740 & 4.0 & 1.7 \\
\hline & Preparatoria & 136,590 & 46,400 & 3.2 & 1.8 & 137,725 & 46,033 & 3.1 & 1.7 \\
\hline & Superior & 64,240 & 22,041 & 3.8 & 1.7 & 73,285 & 25,382 & 3.8 & 1.6 \\
\hline \multirow{7}{*}{2004} & Sin Instrucción & 18,546 & 9,388 & 4.8 & 1.9 & 17,439 & 9,285 & 5.1 & 2.0 \\
\hline & de 1 a 4 años (Primaria) & 23,219 & 10,445 & 4.6 & 1.7 & 22,715 & 11,273 & 4.9 & 1.9 \\
\hline & 5to y 6 to de primaria & 30,188 & 13,641 & 4.3 & 1.7 & 31,488 & 13,660 & 4.6 & 1.8 \\
\hline & Secundaria & 28,760 & 13,406 & 3.9 & 1.6 & 30,604 & 14,655 & 4.1 & 1.6 \\
\hline & 1ro y 2 do de Preparatoria & 43,811 & 18,363 & 4.1 & 1.6 & 44,446 & 18,274 & 4.2 & 1.8 \\
\hline & Preparatoria & 59,166 & 22,073 & 3.8 & 1.6 & 66,445 & 23,964 & 3.7 & 1.6 \\
\hline & Superior & 81,288 & 28,927 & 3.5 & 1.5 & 95,118 & 31,452 & 3.4 & 1.5 \\
\hline \multirow{7}{*}{2006} & Sin Instrucción & 21,040 & 9,834 & 4.7 & 2.0 & 20,115 & 9,764 & 4.7 & 2.0 \\
\hline & de 1 a 4 años (Primaria) & 26,265 & 11,885 & 4.6 & 1.9 & 25,793 & 11,510 & 4.6 & 2.0 \\
\hline & 5to y 6 to de primaria & 31,828 & 13,254 & 4.1 & 1.7 & 33,275 & 13,843 & 4.3 & 1.8 \\
\hline & Secundaria & 41,078 & 16,540 & 4.1 & 1.9 & 38,211 & 15,828 & 4.0 & 1.8 \\
\hline & 1ro y 2 do de Preparatoria & 45,513 & 26,066 & 3.9 & 1.7 & 45,797 & 19,302 & 4.0 & 1.7 \\
\hline & Preparatoria & 60,314 & 21,007 & 3.5 & 1.5 & 62,962 & 21,991 & 3.7 & 1.6 \\
\hline & Superior & 89,372 & 33,659 & 3.3 & 1.6 & 96,059 & 34,369 & 3.2 & 1.6 \\
\hline \multirow{7}{*}{2008} & Sin Instrucción & 21,445 & 10,484 & 5.0 & 3.0 & 22,587 & 11,188 & 4.9 & 3.0 \\
\hline & de 1 a 4 años (Primaria) & 24,905 & 11,533 & 4.6 & 2.7 & 27,148 & 12,081 & 4.6 & 2.7 \\
\hline & 5to y 6 to de primaria & 35,198 & 12,892 & 4.5 & 2.6 & 35,863 & 13,517 & 4.5 & 2.6 \\
\hline & Secundaria & 37,856 & 14,059 & 4.1 & 2.6 & 37,287 & 14,639 & 4.1 & 2.4 \\
\hline & 1ro y 2 do de Preparatoria & 44,545 & 15,501 & 4.1 & 2.5 & 46,954 & 16,314 & 4.1 & 2.4 \\
\hline & Preparatoria & 62,440 & 18,339 & 3.7 & 2.0 & 66,821 & 19,832 & 3.8 & 2.0 \\
\hline & Superior & 94,059 & 24,431 & 3.3 & 1.7 & 104,854 & 26,441 & 3.4 & 1.8 \\
\hline \multirow{7}{*}{2010} & Sin Instrucción & 19,038 & 9,504 & 5.0 & 3.1 & 19,196 & 9,699 & 4.9 & 3.0 \\
\hline & de 1 a 4 años (Primaria) & 23,458 & 10,511 & 4.6 & 2.8 & 24,297 & 11,344 & 4.8 & 2.8 \\
\hline & 5to y 6 to de primaria & 31,007 & 11,986 & 4.3 & 2.6 & 31,587 & 12,202 & 4.5 & 2.6 \\
\hline & Secundaria & 34,598 & 12,090 & 3.8 & 2.2 & 32,160 & 11,997 & 3.9 & 2.2 \\
\hline & 1ro y 2 do de Preparatoria & 39,047 & 14,492 & 4.1 & 2.5 & 39,095 & 14,943 & 4.2 & 2.5 \\
\hline & Preparatoria & 59,116 & 18,362 & 4.1 & 2.4 & 60,156 & 19,183 & 4.0 & 2.3 \\
\hline & Superior & 78,355 & 24,645 & 3.5 & 1.9 & 83,301 & 24,645 & 3.6 & 2.0 \\
\hline \multirow{7}{*}{2012} & Sin Instrucción & 21,015 & 10,670 & 4.7 & 2.6 & 19,300 & 10,080 & 4.7 & 2.5 \\
\hline & de 1 a 4 años (Primaria) & 21,439 & 10,958 & 4.3 & 2.3 & 21,912 & 10,712 & 4.4 & 2.3 \\
\hline & 5to y 6 to de primaria & 29,438 & 13,200 & 4.4 & 2.4 & 29,594 & 13,755 & 4.6 & 2.4 \\
\hline & Secundaria & 63,040 & 25,773 & 4.6 & 2.3 & 52,449 & 23,254 & 4.4 & 2.3 \\
\hline & 1ro y 2 do de Preparatoria & 35,879 & 16,057 & 4.1 & 2.2 & 37,246 & 15,997 & 4.1 & 2.2 \\
\hline & Preparatoria & 50,435 & 19,992 & 4.0 & 2.1 & 55,956 & 21,837 & 4.2 & 2.2 \\
\hline & Superior & 81,410 & 28,751 & 3.8 & 1.9 & 83,108 & 29,068 & 3.8 & 1.9 \\
\hline \multirow{7}{*}{2014} & Sin Instrucción & 17,610 & 9,496 & 5.0 & 3.0 & 19,464 & 10,537 & 5.0 & 2.9 \\
\hline & de 1 a 4 años (Primaria) & 22,384 & 11,215 & 4.7 & 2.6 & 25,202 & 12,073 & 4.7 & 2.6 \\
\hline & 5to y 6 to de primaria & 29,618 & 12,866 & 4.4 & 2.5 & 30,044 & 13,393 & 4.3 & 2.4 \\
\hline & Secundaria & 30,498 & 14,360 & 4.2 & 2.3 & 33,720 & 15,766 & 4.3 & 2.5 \\
\hline & 1ro y 2 do de Preparatoria & 34,629 & 16,081 & 4.1 & 2.3 & 36,799 & 16,342 & 4.2 & 2.4 \\
\hline & Preparatoria & 45,196 & 21,617 & 3.9 & 2.1 & 48,359 & 22,346 & 3.8 & 2.1 \\
\hline & Superior & 78,700 & 29,227 & 3.8 & 2.0 & 81,376 & 29,966 & 3.7 & 2.0 \\
\hline & Sin Instrucción & 19,183 & 11,361 & 4.1 & 2.1 & 21,255 & 11,983 & 4.3 & 2.3 \\
\hline & de 1 a 4 años (Primaria) & 22,862 & 13,102 & 4.3 & 2.3 & 24,977 & 13,663 & 4.3 & 2.3 \\
\hline & 5to y 6 to de primaria & 27,716 & 13,877 & 4.1 & 2.1 & 29,986 & 14,417 & 4.1 & 2.1 \\
\hline 2016 & Secundaria & 31,944 & 16,037 & 3.8 & 2.0 & 34,234 & 16,591 & 3.8 & 2.0 \\
\hline & 1ro y 2 do de Preparatoria & 34,174 & 16,336 & 3.7 & 1.9 & 36,268 & 16,964 & 3.8 & 2.0 \\
\hline & Preparatoria & 49,443 & 21,941 & 3.6 & 2.0 & 50,321 & 21,682 & 3.6 & 1.9 \\
\hline & Superior & 76,094 & 30,922 & 3.3 & 1.7 & 76,323 & 30,704 & 3.3 & 1.7 \\
\hline
\end{tabular}

Nota: Se utilizaron los mismos grupos (grupo de edad y año de nacimiento del jefe de familia) para los promedios utilizando los factores de expansión para cada levantamiento de la ENIGH y los obtenidos en la construcción del Panel Sintético.

Fuente: Estimación propia con datos del INEGI-ENIGH 2008-2016. 